This item was submitted to Loughborough's Research Repository by the author.

Items in Figshare are protected by copyright, with all rights reserved, unless otherwise indicated.

\title{
Imprecise visual feedback about hand location increases a classically conditioned pain expectancy effect
}

PLEASE CITE THE PUBLISHED VERSION

https://doi.org/10.1016/j.jpain.2021.01.004

PUBLISHER

Elsevier

VERSION

AM (Accepted Manuscript)

\section{PUBLISHER STATEMENT}

This paper was accepted for publication in the journal The Journal of Pain and the definitive published version is available at https://doi.org/10.1016/j.jpain.2021.01.004.

\section{LICENCE}

CC BY-NC-ND 4.0

\section{REPOSITORY RECORD}

Chaves, Thais Cristina, Tasha R Stanton, Ashley Grant, Brian W Pulling, Victoria J Madden, Roger Newport, and G Lorimer Moseley. 2021. "Imprecise Visual Feedback About Hand Location Increases a Classically Conditioned Pain Expectancy Effect". Loughborough University. https://hdl.handle.net/2134/13720747.v1. 
Short Running Title: Disrupted visual hand feedback increases pain expectancy

Thais Cristina Chaves ${ }^{1,2}$, Tasha R. Stanton ${ }^{1}$, Ashley Grant ${ }^{1}$, Brian W. Pulling ${ }^{1}$, Victoria

1. IIMPACT in Health, University of South Australia, Adelaide, Australia

2. Department of Health Sciences, Ribeirão Preto Medical School, University of São Paulo - USP, Brazil (Corresponding author)

3. Department of Anaesthesia and Perioperative Medicine and Department of Psychiatry and Mental Health, Neuroscience Institute, University of Cape Town, South Africa

4. School of Psychology, University of Nottingham, Nottingham, UK; School of Sport, Exercise and Health Sciences, Loughborough University, Leicestershire, UK.

Address for correspondence:

Thais Cristina Chaves - Department of Health Sciences, Ribeirão Preto Medical School, University of São Paulo - USP

Avenida Bandeirantes 3900 CEP: 14049-900, Ribeirão Preto (SP) Brazil

e-mail: chavestc@,fmrp.usp.br

\section{Research funding}

GLM is supported by a Leadership Investigator Grant from the National Health \& Medical Research Council of Australia ID 1178444. TCC was supported by FAPESP (São Paulo Research Foundation) - grant process 2016/15365-2. TRS was supported by Career Development Fellowship from the National Health \& Medical Research Council of Australia ID 1141735. VJM is supported by an Innovation postdoctoral research fellowship from the National Research Foundation of South Africa. BWP's PhD is supported by a University President's Scholarship (University of South Australia).

\section{Disclosures}

TRS received support from Eli Lilly Ltd for travel expenses; this is unrelated to the present topic. GLM has received support from ConnectHealth UK, AIA Australia, SwissRe, Gallagher Bassett, Kaiser Permanente, Workers' Compensation Boards in Australia, Europe and North America, the International Olympic Committee, Port Adelaide Football Club, Arsenal Football Club, Pfizer, Seqirus and various professional organisations and learned societies. He receives royalties for several books on pain and speakers' fees for talks on pain and rehabilitation. VJM receives speakers' fees for talks on pain and rehabilitation. RN and TCC discloses no conflict of interest. BWP has received personal fees for scientific writing for Cosmos Magazine, and honoraria from Elsevier for contributing to a textbook on an unrelated subject. He is a volunteer for PainChats LLC., and for Pain Revolution. 
Abstract: We tested the hypotheses that rendering sensory input about hand location 44 imprecise increases a classically conditioned pain expectancy effect, increases 45 generalization of the effect to novel locations and reduces extinction of the effect. Forty 46 healthy volunteers performed movements with their right hand along predefined paths. 47 Each path passed through two locations that were defined as either (i) the conditioned 48 stimulus (CS+; paired with a painful unconditioned stimulus - UCS), and (ii) unpaired 49 (CS-). During acquisition phase, participants watched their hand as they moved it. 50 Participants were randomly allocated to an Imprecise group (IG), for whom visual whe for whom vision was not disrupted. In the test phase, participants moved their hands to five locations - the CS+, CS- and three locations that lay between the two ('Generalisation stimuli'). Our first hypothesis was reported - pain expectancy was greater at the $\mathrm{CS}+$ location in the IG than in the PG (6.9 [SD=1.9] vs 5.4 [SD=2.5], $\mathrm{p}=0.02$ ). Pain expectancies generalised to novel locations similarly in both groups and there was no difference in extinction between groups. Our primary hypothesis was supported but our subsequent hypotheses were not.

Perspective: We conditioned pain expectancy at a certain location of one hand, even though most participants were unaware of the contingency. Conditioned pain expectancy was greater when sensory information about location was less precise. This adds support to the possibility that associative learning may play a role in the progression of an acute pain episode to a more generalized pain disorder.

Key words: Classical Conditioning, imprecise stimulus, illusion, pain expectancy, hand location 


\section{Introduction}

82 Chronic musculoskeletal pain is a major health problem with a one-year prevalence of $25 \%$ to $36 \%$ in the general population ${ }^{35}$. Costs related to persistent pain in the United States of America are between $\$ 560$ and $\$ 635$ billion annually ${ }^{18}$. Many persistent pain states are not associated with ongoing tissue pathology, an originally perplexing observation that is now explained by functional changes in the nociceptive system and brain $^{79-81}$. Broadly speaking, these functional changes may be considered learning; stimulus-response profiles change such that stimuli that are not normally painful come to evoke pain, a situation termed allodynia, and normally painful stimuli come to evoke more pain, a situation termed hyperalgesia ${ }^{79}$.

91 The vast majority of research has considered this learning in the nociceptive system to 92 reflect non-associative learning, whereby synaptic efficacy is enhanced by repeated signalling and the consequent long-term potentiation of the post-synaptic neurone results in 'central sensitisation'80. However, the vast majority of persistent pain states cannot be explained by this central sensitisation, a reality that led to a radical updating of the idea of central sensitisation to a clinical observation of allodynia and hyperalgesia in response to stimuli delivered outside the body area that was initially affected ${ }^{81}$. Even still, many pain states involve allodynia to a range of non-noxious somatosensory and, in fact, to non-somatosensory cues ${ }^{1,59,24}$, pointing to the possibility that associative learning may also contribute to persistent pain.

101 The possibility that associative learning, or classical conditioning processes, might 102 contribute to persistent pain, is widely endorsed clinically ${ }^{45}$, even though supportive empirical data from humans has only emerged recently and inconsistent results between experiments point to a complex picture ${ }^{3,27,32,42}$. It is critical to clearly differentiate this notion from that of associative learning of pain-related fear, for which there is a vast 
106 literature ${ }^{13,75,76}$. In that work, pain is considered an unconditioned stimulus (US) and

107 fear the conditioned response (CR). Here, however, nociception is considered the US and 108 pain the $\mathrm{CR}^{60}$.

109 That non-noxious signals might come to evoke or magnify pain would be predicted by 110 contemporary theories of brain function ${ }^{31,78}$ and the biopsychosocial model ${ }^{17}$ of pain $^{37}$,

$11{ }^{38}$, the latter of which posits that pain emerges from the interplay between contributors

112 from across biological, psychological and environmental or contextual domains [see

113 Moseley and Butler ${ }^{55,56}$, for extensive reviews]. Empirical data are also supportive. For

114 example, delivering a noxious cold stimulus with auditory or visual cues associated with

115 heat evokes intense pain and often a feeling of intense heat, but delivering an identical

116 noxious cold stimulus with auditory or visual cues associated with cool evokes less

117 intense pain and usually a feeling of cold ${ }^{2,55}$. Also, in people with neck pain, manipulating

118 visual feedback during a head rotation task shifts the point at which they report the onset

119 of pain, in a direction-specific manner ${ }^{27}$.

120 These converging lines of discovery led to and support the proposal known as the

121 'Imprecision Hypothesis $(\mathrm{IH})$ of chronic pain' ${ }^{60}$. It predicts that associative learning 122 contributes to the progression of an episode of acute pain to a generalised pain disorder 123 via over-generalisation of the conditioned response. Several studies have now supported 124 that allodynia and hyperalgesia can be induced experimentally via classical conditioning 125 procedures $3,25,26,32,46,49,71$ as suggested by IH. Key to the IH is the notion that imprecise 126 encoding of the multisensory CS that routinely coincides with the nociceptive input leads 127 to over-generalisation of any classical conditioning effect ${ }^{60}$. Imprecise encoding might 128 occur under situations in which sensory channels that dominate the multisensory CS are 129 disrupted. Such disruptions have been documented in people with persistent pain ${ }^{9,58,68}$. 130 Relevant here are contemporary ideas in predictive processing, wherein perception is 
131 argued to result from the integration of incoming sensory data and top-down predictions

132 based on internal, generative models ${ }^{29}$. In contexts of precise or ecologically important

133 predictions and imprecise sensory inputs, perceptions can deviate from the actual state of

134 the world ${ }^{64}$. That is, in contexts of predictions that are fundamentally important to

135 protection - for example pain - alongside imprecise sensory inputs, perceptions can

136 deviate toward increased probability of pain. We have previously shown pain expectancy

137 - the likelihood that pain will occur - to be higher when a CS occurred in anatomical areas

138 of low somatosensory precision (back) than when it occurred in areas of high

139 somatosensory precision $^{26}$. However, that result leaves open the possibility that

140 differences in somatosensory precision between the two anatomical areas did not

141 underpin the difference in pain expectancy.

142 Here we interrogated Pain Expectancy - the probability of pain - under different

143 conditions of sensory precision, induced by the MIRAGE illusion system ${ }^{66}$. Our first

144 hypothesis (H1) was that imprecise sensory input during conditioning would result in

145 greater expectation of pain (the 'CR') at the CS+ location. Our secondary hypotheses

146 were that (i) generalization of the conditioned pain expectancy would be greater, and (ii)

147 extinction of the effect slower, when sensory input during acquisition was less precise.

149 Methods

150 Overview of the Procedure

151 Forty participants underwent a differential classical conditioning experiment. First, 152 participants were submitted to a calibration procedure to determine the individual 153 intensity of electrical aversive stimulation delivered. Afterwards, using the MIRAGE 154 system, participants were trained on how to move their right hand along paths (without 155 aversive stimulation) while they watched a real-time video of their hand beneath the 
156 mirror (see Figure 1). The MIRAGE illusion system has a camera, a customized software

157 program, a monitor and mirrors to allow participants to watch a real-time video of their

158 hand beneath the mirror, from the same perspective and in the same spatial location as if

159 they were viewing the right hand directly.

160 During the acquisition phase, participants were randomly allocated to an Imprecise group

161 - vision was disrupted to right or left - or a Precise group - vision was not disrupted. To

162 manipulate the accuracy of visual input of hand location (imprecision), the MIRAGE

163 system applied a shift in the apparent (i.e. visually encoded) hand location of 30 to 50

$164 \mathrm{~mm}$ (twice at each offset, order randomised), while the participants moved their right

165 hand on the paths randomly determined. The paths were nonlinear trajectories starting

166 and finishing always on the bottom right of the MIRAGE board and should include two

167 locations: one on the far upper left side and one on the far upper right side of the

168 movement path. One location was the conditioned stimulus $(\mathrm{CS}+)$, paired with an

169 electrical aversive unconditioned stimulus (UCS). Another location was the CS- and was

170 unpaired. The locations that lay between CS+ and CS- in the upper part of the MIRAGE

171 board, but outside the predefined movement paths, were the generalization stimuli (GSs).

172 In $50 \%$ of trials $(\mathrm{n}=6)$, when the hand crossed the CS+, the UCS was delivered to the 173 hand.

174 In test phase, participants reported how likely they expected pain if their hand reached

175 five specific locations (imagery task) marked with dots, including CSs and three novel

176 locations (GSs - not available during acquisition). The locations were projected on the

177 MIRAGE screen. The procedure was repeated randomly four times for each location (just

178 one location was visible on the screen per trial) and Pain Expectancy ratings were

179 obtained for each location. 
180 The extinction phase required participants to move their hand along paths (12 times) that

181 included the CS+ and CS- location, but no UCS was delivered. In each extinction trial, a

182 movement path was shown on the screen, but this time the CS+ and CS- location were

183 marked with dots (six trials for CS+ and six trials for CS-). Participants provided Pain

184 Expectancy ratings as they had in the test phase, and then performed the movement as

185 they had in the acquisition phase, but the UCS was never presented.

\section{Participants}

188 We calculated our sample size considering mean and standard deviations of Pain

189 Expectancy for CS+ between groups, from the first 5 participants we enrolled in each

190 group $(\mathrm{n}=10)$. We powered to detect a medium between-group effect size (Cohen's

$191 \mathrm{f}=0.43$ ); power of $90 \%, \alpha=0.05$, correlation between measures $=0.40$; repeated measures

192 between factors (G*power, Faul, Erdfelder, Buchner \& Lang; Institute of Psychology,

193 University of Duesseldorf, Version 3.1, Germany). Accordingly, we required 17

194 participants per group. To allow for withdrawals and technical errors, we aimed to recruit

19520 in each group.

196 The volunteers were recruited through flyers, social media and word of mouth at the

197 University of South Australia. This study enrolled right-handed males and females (with

198 normal or corrected-to-normal vision), aged between 18 and 50 years old. All eligible

199 participants signed a consent form and received an honorarium of AUD \$20 per hour.

200 Participants were not aware of the real aim of the study. Specifically, they were unaware

201 that: i) they could experience a visual illusion during the acquisition phase of the study,

202 and ii) that it was a classical conditioning study. However, we provided them with the

203 information that they would receive a noxious stimulation to investigate the relationship

204 between learning about movement and pain. The experimental protocol described that it 
was a deception study and was approved by the Human Research Ethics Committee from

206 University of South Australia (HREC; protocol number: 200706).

207 The following exclusion criteria were applied: i) Neurological diseases (Cerebral 208 paralysis, cerebrovascular accident or sequelae, epilepsy, Multiple Sclerosis, Parkinson's 209 Disease, post-Herpetic neuralgia) or any history of trauma in the hand; ii) Chronic 210 disorders (diabetes), vascular problems or chronic pain (pain which has lasted longer than

21112 weeks and is present on most days); iii) History of chronic pain within the last 6 212 months, iv) Pregnancy, v) Acute pain (at any site); vi) Hand pain or recent hand injury 213 within the previous 12 weeks; vii) diagnosed psychiatric disorder; viii) any skin 214 sensitivity (dermatitis, psoriasis and eczema); ix) heart problems or a pace maker; and $\mathrm{x}$ ) 215 surgical pins or plates or metal-based tattoos in the hands.

216

217 Classical conditioning: stimuli and manipulation check

218 This experiment used a classical conditioning procedure designed to create an association 219 between the participant's hand being at a certain location (CS+) and the occurrence of a 220 aversive stimulus (US).

221

\section{Conditioning and generalisation stimuli}

223 A set of three different hand movement paths was created such that each path passed 224 through two defined locations: one on the far upper left side and one on the far upper right 225 side of the movement path (Figure 1A). One of the locations was defined as CS+ and was 226 paired with an aversive electrical stimulus to the hand; the other was defined as CS- and was unpaired. The allocation of location (left/right) to CS was counterbalanced between participants within each group. Three distinct locations that lay between the two CSs, but outside the predefined movement paths, were defined as generalisation stimuli (GSs). The 
230 GSs were numbered consecutively, with the numbers increasing with distance from the

231 CS+. A picture illustrating the board with the five locations (marked with dots) and one 232 of the paths adopted in the study can be found in the (Figure 1B).

233

\section{Unconditioned stimulus}

235 The UCS was an electrocutaneous stimulation (square wave pulse of duration 100ms, $236400 \mathrm{~V}$ ) applied to the back of the hand in the space between the first and second metacarpal 237 bones. A pushpin-type electrode resembling that of Inui et al. ${ }^{30}$, comprising a concentric 238 anode and a blunt pin-type cathode in the center, was manufactured in house and attached 239 to the skin with a circular adhesive sticker. Electrical current was passed across the 240 electrode using a Digitimer device (DS7AH, SDR Scientific, Power: 12 Va, Freq: 47-63

241 Hz, Hyde Way Welwyn, Garden City, UK) which was manually controlled by a 242 researcher.

\section{Contingency awareness}

245 Immediately after the end of the experiment, participants responded to four questions that 246 aimed to investigate associations formed between locations, movement paths and painful 247 stimulation or visual distortion during the experiment: (i) "in which position did you feel a painful stimulation?”, (ii) "in which position(s) did you perceive visual distortion?",

249 (iii) "in which path(s) did you feel a painful stimulation?" and (iv) "in which path(s) did 250 you perceive a visual distortion?". To help participants to answer the questions, the 251 pictures of the locations and paths were projected on the screen. 
254 Participants were randomly allocated to one of two groups - Precise or Imprecise. The

255 Precise group undertook the classical conditioning procedure with normal visual input.

256 The Imprecise group undertook the classical conditioning procedure with modified visual

257 input that shifted the seen position of the hand (Figure 1B). To manipulate the visual input

258 for the Imprecise group, we used the MIRAGE illusion system (Figure 1A), which has a

259 camera, a customized Labview software program (2010 National Instruments $\left.{ }^{\circledR}\right)$, a

260 monitor and mirrors to allow participants to watch a real-time video of their hand beneath

261 the mirror, from the same perspective and in the same spatial location as if they were

262 viewing the right hand directly (Figure 1B). The MIRAGE system was used for all

263 participants, but the illusion was applied for the Imprecise group only. Participants sat on

264 a chair and placed their hand into the lower level of the MIRAGE box, to lie on a

265 horizontal board. Thus, when a participant looked down towards their hand, they saw a

266 live footage of their right hand. A partition and bib secured around participants' necks

267 prevented the participant from seeing their real hand. The participant's limb appeared in

268 the same spatial location and from the same perspective as it would if they were viewing

269 the limb directly (Figure 1B).

270 To manipulate the accuracy of visual input of hand location, the MIRAGE system applied

271 a shift in the apparent (i.e. visually encoded) hand location of $30 \mathrm{~mm}, 40 \mathrm{~mm}$ or $50 \mathrm{~mm}$ to

272 the right or left (twice at each offset, order randomised), while the participant performed

273 movements on paths during the acquisition phase (See Figure 1). The magnitude of the

274 shift was determined through pilot testing (Supplementary Method S1 and Figure S2).

275 It was necessary to prevent the participant from updating or correcting the hand position

276 to match visual estimates [proprioceptive recalibration ${ }^{12}$ ] as they performed the

277 movements during the acquisition and extinction phases. To achieve this, a black

278 rectangle obscured $70 \%$ of the board on the screen, so that the participant only saw his/her 
hand once it reached the CS+ or CS- location. This limited the opportunity to adjust the

280 movement path on the basis of visual input.

Visual illusion manipulation check

283 In order to verify that the actual and seen hand locations during painful stimulation had

284 differed within the Imprecise group, we measured the seen and actual positions of the 285 hand in the space at the moment when the painful stimulation was delivered 286 (Supplementary methods - Figure S3). The tracking system (Labview customized

287 software) was used to calculate the distance between the tip of the middle finger and the 288 lateral border of the screen for the Precise group. For the Imprecise group, both the actual 289 and the seen distances between the tip of the middle finger in relation to the lateral border 290 of the screen were considered (Supplementary methods - Figure S3). The difference 291 between the actual and seen distance was used to identify the "real visual shift" achieved 292 for the Imprecise group.

293 The program ImageJ (Rasband, W.S., ImageJ, U. S. National Institutes of Health, 294 Bethesda, Maryland, USA, https://imagej.nih.gov/ij/, 1997-2016) was used to obtain the 295 values in millimeters. To clarify - the true hand location at which the noxious stimulus 296 was delivered was intended to consistently vary from the visually encoded location of the 297 hand in the Imprecise Group via illusion. The above measures were used to confirm 298 whether this occurred.

300 Awareness of manipulation of visual input

301 At the end of the study, before disclosing the real nature of the study, participants

302 indicated how much they agreed (or not) with four statements: "I felt as if I was looking 303 at my own hand", "I felt as if I was causing the movement I saw"; "Sometimes, I felt there 
was something wrong during some movements; "Sometimes, I felt like an incongruence

305 between my hand position and the visual feedback about my hand". The response options

306 were Likert-type scale ranging from: strongly agree, agree, neither agree or disagree,

307 disagree and strongly disagree. Although only the Imprecise group had received the visual

308 manipulation, all participants responded to these questions.

\section{Outcomes and Questionnaires}

311 Primary hypothesis outcome: Pain expectancy ratings for the CS+ location during test

312 phase. Secondary hypothesis outcomes: Pain expectancy ratings at the remaining

313 locations during test phase, and Pain Expectancy ratings at each location during extinction

314 phase. The question and anchors in both cases were: 'How likely do you think it is that

315 you will receive a painful stimulation?', with 0 meaning "not at all likely", and 10

316 meaning "extremely likely".

\section{Sensation and pain intensity}

319 To assess discomfort and pain during the calibration procedure, participants rated all

320 stimuli using the Sensation and Pain Rating Scale (SPARS) ${ }^{43}$ which has anchors of "no sensation" (-50), “the exact point at which what you feel transitions to pain" $(0)$, and "most intense pain you can imagine", $(+50)$. The SPARS performs well in the experimental context and overcomes limitations in scale range inherent to conventional

324 pain rating scales ${ }^{43}$. We asked participants to rate their experience on the appropriate side

325 of the scale, with -50 meaning 'no sensation', +50 meaning 'the most intense pain you can imagine' and 0 meaning 'the exact point at which what you feel transitions to pain'.

327 The written explanation of the SPARS emphasizes that ratings between -50 and 0 reflect 328 a non-painful experience and ratings between 0 and 50 reflect a painful one. 
330 Questionnaires administered at the baseline

331 To characterize the sample, participants completed the Positive and Negative Affect

332 Schedule (PANAS) ${ }^{11}$ and the State-Trait Anxiety Inventory (STAI) ${ }^{67}$. Both have

333 acceptable internal consistency $(\alpha>.85)$, construct validity and structural validity ${ }^{11,67}$.

334 Low positive affect and high trait anxiety have been linked to reduced extinction in fear 335 conditioning experiments ${ }^{52}$.

336

337 Questionnaire administered at the end of the experiment

338 The extent to which participants engaged in catastrophic thinking during the application

339 of individually calibrated painful stimuli $^{16}$ was assessed using the Catastrophizing

340 Questionnaire. There are no widely accepted measures of catastrophizing in response to

341 experimental pain stimulation. This questionnaire is a modified version of the Pain

342 Catastrophizing Scale ${ }^{70}$. The wording of 6 items were modified to represent the three

343 primary dimensions of catastrophizing in the context of laboratory procedures:

344 rumination, magnification, and helplessness. Immediately after undergoing the

345 experimental procedure, participants rated the degree of catastrophizing (during the

346 painful stimulation) using the 6-item scale. The response options were: 0 (not at all), 1

347 (to a slight degree), 2 (to a moderate degree), 3 (to a great degree) and 4 (all the time).

348 Catastrophizing scores were obtained by summing the scores on the 6 items (maximum

349 possible score: 24). Cronbach's alpha for the scale was 0.87 , suggesting a high degree of 350 internal consistency.

352 Procedure 
353 In the first contact with the volunteers (email or phone call), they were screened for right

354 hand dominance (using the Edinburgh Handedness Inventory ${ }^{63}$ and exclusion criteria 355 were applied. Upon arrival at the laboratory, participants completed the informed consent

356 form, filled in the baseline questionnaires and we obtained anthropometric data (weight,

357 height and age). The skin on the dominant hand was exfoliated and cleaned with alcohol,

358 and the electrode was taped to the skin. Micropore hypoallergenic medical tape fixed the

359 cable to the dorsal surface of the wrist.

360 Participants were then allocated to one of the groups (Precise or Imprecise) via concealed

361 randomisation. Also, the $\mathrm{CS}+$ location and the sequence of administration of the $\mathrm{CS}+$ and

362 CS- were determined via a concealed simple randomization from five pre-defined

363 possible sequences. This procedure guaranteed that half the participants would receive

364 the US on each side of the board in each group. The randomization order was predefined using study randomizer online (https://studyrandomizer.com/).

366

\section{Calibration procedure}

368 We used an established calibration procedure to determine the intensity of electrical

369 stimulation needed to elicit a self-report of moderate pain, defined as 'painful and requires

370 effort to tolerate' and corresponding to a SPARS rating of between +25 and $+35^{71}$. First,

371 participants were submitted to the following electrical stimuli with a 30 -second inter-

372 stimulus interval: $1 \mathrm{~mA}$ (presented twice), $2 \mathrm{~mA}$ (twice), 4mA (twice), $6 \mathrm{~mA}$ (once) and

$3738 \mathrm{~mA}$ (once). During this time, if any impedance occurred, then the electrode was

374 repositioned until the impedance no longer occurred and the procedure was restarted.

375 Next, a series of electrocutaneous stimuli of increasing intensity was administered to the

376 participant's hand, starting with $2 \mathrm{~mA}^{71}$. The final higher electrical stimulus, in which the

377 participant rated four in six trials as painful and greater than +25 using the SPARS, was 
378

379

380

381

382

383

384

385

386

387

388

389

390

391

392

393

394

395

396

397

398

399

400

401

used as the US during Acquisition phase. After three minutes of rest, the acquisition phase has started.

\section{Training phase}

After calibration, participants sat at the MIRAGE and placed their right hand inside the lower level of the MIRAGE box (Figure 1A), such that they could see the real-time footage of their hand. First, each pre-defined movement path was projected onto the MIRAGE screen (mirror reflecting the monitor), and the participant memorized the path, including the direction of movement, as cued by visible arrows. Participants practised performing a 15 -second movement of their hand along the path in a clockwise or counterclockwise direction, 2 or 3 times per path, with feedback given to improve accuracy. The time was controlled by a chronometer. To maintain the accuracy of the movement tracking system, the prescribed position of the hand was with the fingers pointing forward, the hand held flat, the thumb in adduction and the fingers held together. Participants were also instructed to begin the movement after verbal cuing, to keep the hand within the visible area of the screen, and to return to the start position after the end of each movement. During training phase, the participants on both groups were not submitted to any illusion.

\section{Acquisition phase}

The Acquisition phase consisted of 12 trials. Each trial involved one cued movement along a prescribed movement path. There were three different possible movement paths (three in clockwise and three in counter-clockwise direction), each presented twice in a variable counterbalanced fashion. Every prescribed movement path passed through the 
402 CS+ and CS- locations but did not pass through any of the GSs locations (Figure 2 and 403 Supplementary files - Figures S4 and S5).

404 In $50 \%$ of Acquisition trials, the US was delivered at the moment when the participant's 405 hand crossed the CS+ location. Participants received CS-UCS parings just $50 \%$ of the 406 trials (6 times), because in all the trials participants had to move on paths crossing the

407 CS + position. This was based on pilot trials. If we administered the aversive stimulus 408 every time the participant crossed the CS+ position, there was a risk that participants 409 would pair 'movement' and 'pain', rather than a particular location and pain, thus 410 confounding our intended manipulation .

411 Participants were requested to move the hand along each path spending approximately 41215 -seconds as they were trained. This time interval was determined during the pilot study 413 as a comfortable speed. Feedback was provided at the end of each trial, with instructions 414 to move fast or slow. We controlled the time spent and gave instructions to improve 415 accuracy. The time was controlled by a chronometer.

416 For the Imprecise group, the visual image of the hand was offset by $30 \mathrm{~mm}, 40 \mathrm{~mm}$ or

$41750 \mathrm{~mm}$ to the right or left (two trials of each, order randomised). A snapshot was captured 418 on the researcher's screen and stored at each moment when the hand reached the CS 419 locations mandatory in that trial (i.e.: if the trial was related to $\mathrm{CS}+$, the snapshot was 420 obtained just for such hand position) so as to later confirm the visual shift in the hand 421 position when the electrocutaneous stimulus was administered (Supplementary methods 422 Figure S6).

423 To avoid proprioceptive recalibration (for further details see the section "The Precise and 424 Imprecise conditions") the participants were required to move their hand along the paths 425 without visual information until they reached the position of the CS+. A rectangular zone 426 (10 cm wide, $16 \mathrm{~cm}$ long) at the location of each CS was designated as a 'target zone' in 
the customized Labview tracking system. During the acquisition phase, as soon as the

428 tracking system detected that a participant's hand had entered the CS+ "target zone", the

429 UCS was delivered (Supplementary methods Figure S6). After three minutes of rest, we

430 started the test phase.

431

432 Test phase

433 The test phase involved no presentation of CS or UCS, was identical for both groups, and

434 involved no visual illusions. In each of 20 trials, the participant was shown one of the five

435 locations (marked with dots) corresponding to Stimulus type: both CSs and three

436 intermediate positions between CS + and CS- (GSs positions). Then, they were invited to

437 report "... how likely do you think is that you will receive a painful stimulation?" if they

438 move their hand to a specific location illustrated on the screen (Figure 2).

439 Each location was presented in 4 trials, and the order of trials was chosen by lot (five sequences pre-defined) (Supplementary file S2).

441

442 Extinction phase

443 The extinction phase started just after the test phase ( 2 minutes of rest). The extinction 444 phase consisted of 12 trials, 6 trials for CS+ location and 6 trials for CS- location, was 445 identical for both groups, and involved no visual illusion. In each extinction trial, a 446 movement path was shown on the screen, but this time the CS+ and CS- locations were 447 marked with dots. The volunteers were invited to move on the same paths performed 448 during acquisition. Participants provided Pain Expectancy ratings as they had in the test 449 phase, and then performed the movement as they had in the acquisition phase, but the US 450 was never presented (Figure 2).

451 
453 Finally, participants completed the catastrophizing questionnaire, responded to the 454 conditioned stimuli recognition test, were asked about contingency awareness and visual manipulation awareness, and, finally, the real nature of the study was explained.

\section{Statistical Analysis}

458 The primary analysis for this study used Pain Expectancy ratings provided during the test phase. We used Mixed-Design ANOVAs to compare expectancy ratings across locations and between groups. Our primary aim was to compare Pain Expectancy for CS+ between

461 groups (H1). Our secondary aim was to compare pain expectation generalization (H2)

462 and delay in extinction (H3), within and between-groups.

463 To test the primary hypothesis, we ran a Mixed-Design ANOVA considering Pain

464 Expectancy to CS+ and CS- (4 levels) as repeated measures and Groups (2 levels: Precise or Imprecise) as between-subjects factor. Considering test phase results, we ran another Mixed-Design ANOVA to test our secondary hypothesis (H2), in which GS1, GS2 and

467 GS3 (4 levels) were the repeated measures and Groups (2 levels: Precise or Imprecise) as 468 the between-subjects factor. With the aim to test our secondary hypothesis (H3), we also performed a Mixed-Design ANOVA with Pain Expectancy to CS+ and CS- (6 levels) as repeated measures and Groups (2 levels: Precise or Imprecise) as between-subjects factor.

471 In addition, separate repeated measures ANOVAS on both the Precise and Imprecise 472 groups were conducted to test H2, considering the mean Pain Expectancy on test phase 473 trials (average of Pain Expectancy across trials) for each stimulus (CS, GS1, GS2, GS3, 474 CS-, five levels). And two separate repeated measures ANOVAs on both Precise and 475 Imprecise groups to investigate within-subject effect of trials on Pain Expectancy during 476 extinction phase. 
477 The Kolmogorov-Smirnov test and the M Box test were applied to assess the normality

478 of the distribution and homogeneity of variance of our data, respectively. The Mauchly's

479 test was used to verify the assumption of sphericity. To compare the means between the

480 different levels of independent variables (pairwise comparisons), Bonferroni test

481 (correction) was used to control for type I error. The comparison between the Precise and

482 Imprecise groups for anthropometric data and psychosocial variables was carried out

483 using a simple multivariate ANOVA $(\mathrm{p}<0.05)$ considering Group as the between-subjects

484 factor. Also, the chi-square test was used to analyze percentage values. Data were 485 analyzed using Statistical Package for the Social Sciences (SPSS, Chicago, IL) 22 for 486 Macbook and were expressed as estimated mean and standard deviation (SD).

487

\section{$488 \quad$ Results}

489 Fifty participants volunteered. Four were excluded: one participant reported isopropyl 490 alcohol allergy, one participant reported a rare skin disease (Dermatographic urticaria), 491 one participant reported a heart disorder (prolapsed mitral valve) and one participant 492 reported Raynaud's syndrome. Additional four volunteers were excluded after changes 493 to the experiment (pilot study) and two participants were excluded from analysis after the 494 procedure because they did not report moderate pain on the SPARS (score of at least 25/50) even when exposed to a high intensity noxious stimulation (more than $60 \mathrm{~mA}$ ) 496 suggesting a possible nerve accommodation/habituation phenomenon ${ }^{28}$. Finally, 40 497 participants ( $n=20 /$ group) were included in the study. Baseline questionnaire results are 498 provided in Table 1. No differences were observed between groups for age. The Precise 499 group had a higher body mass index (BMI) than the Imprecise group, however, both 500 groups showed a BMI score of normal weight (18.5-24.9). Nevertheless, we investigated

501 the effect of the BMI as a confounding variable on the Pain Expectancy ratings. 
504 We calculated the actual and seen hand distances $(\mathrm{mm})$ from the top middle finger to the 505 closest border of the picture (screen) in the exact moment the participants received the 506 painful stimulation. The mean difference in distance [actual hand - seen hand] was similar 507 to the shift intended, except when the leftwards shift of $50 \mathrm{~mm}$ was administered to the 508 group with $\mathrm{CS}+$ on the right side of the board, when the actual shift was $-42 \mathrm{~mm}$ 509 (Supplementary results - Table 1S). Regardless, these findings confirm that in the 510 Imprecise condition, a difference between seen and actual hand locations was always 511 introduced, reflecting a consistent imprecision in sensory input as intended.

\section{General statistics}

514 The assumption of normality was not met for all variables: the Kolmogorov-Smirnov test 515 showed a significant deviation from normality $(\mathrm{p}<0.05)$ for two ratings of two locations 516 (GS2, CS-) during test phase. However, the literature suggests that the F-test is robust, in 517 terms of power, tolerating violations of normality even with very small sample sizes ${ }^{33}$.

518 Also, because the assumption of sphericity was violated (significant Mauchly's test), we 519 adopted the results as recommended according to the epsilon boundary of $0.75^{19}$. Huynh520 Feldt-corrected results were adopted for both test and extinction phases $(\varepsilon>0.75)$.

522 Pain expectancy during test phase

523 To test our first hypothesis (H1), we ran a mixed model ANOVA which showed a main

524 effect of Group on Pain Expectancy at the $\mathrm{CS}+\left(\mathrm{F}_{(1,692,64,278)}=4,95, \mathrm{p}<0.01, \eta_{\mathrm{p}}{ }^{2}=0.12\right)$. We

525 ran the analysis twice, with and without adjustment for seven predictors: positive affect, 526 negative affect, anxiety, catastrophising, pain rating obtained during calibration, sex and 
527 BMI. None of the predictors showed a significant effect on Pain Expectancy and did not 528 change the main effect of Group on Pain Expectancy, hence we considered the results of 529 the analysis with no adjustment. In pairwise comparison, the Imprecise group showed a 530 higher Pain Expectancy for CS+ than the Precise group did (6.9 vs. 5.4, p=0.03) which 531 upheld our first hypothesis (H1) (Table 2).

532 Between-group comparison on Pain Expectancy at each of the generalization stimuli was conducted with the aim to test our secondary hypothesis (H2). There was no difference between-groups for GS1 $\left(\mathrm{F}_{(1,38)}=0.005, \mathrm{p}<0.92, \eta_{\mathrm{p}}{ }^{2}=0\right)$, GS2 $\left(\mathrm{F}_{(1,38)}=0.36, \mathrm{p}<0.54, \eta_{\mathrm{p}}{ }^{2}=\right.$ $0)$ and GS3 $\left(\mathrm{F}_{(1,38)}=0.23, \mathrm{p}<0.63, \eta_{\mathrm{p}}{ }^{2}=0\right)$ (Table 2$)$. We also ran separate repeated measures ANOVA for Precise and Imprecise groups to investigate within-subject differences in Pain Expectancy between different stimuli (across locations), particularly differences between CS+ and the GSs. We showed a significant effect of stimulus location (conditioned or generalization stimulus) on Pain Expectancy for both the Precise

$540 \quad\left(F_{(1,6836,750)}=3.8, \mathrm{p}=0.01, \eta_{\mathrm{p}}{ }^{2}=0.23\right)$ and the Imprecise groups $\left(\mathrm{F}_{(3,190,41,47236,750)}=3.77\right.$,

$541 \mathrm{p}=0.01, \eta_{\mathrm{p}}{ }^{2}=0.22$ ). Pairwise comparisons (Bonferroni correction) showed a lower Pain Expectancy at GS3 and CS- than at CS+ for both groups (Table 3). The pain expectancies

543 for each group and each location showed a gradual decrease in Pain Expectancy as the 544 distance from the CS+ increased (Table 3).

546 Pain expectancy during extinction phase

547 To investigate our third hypothesis, we ran a Mixed Model ANOVA on Pain Expectancy 548 ratings during extinction phase. This ANOVA showed no main effect of Group on Pain 549 Expectancy at $\mathrm{CS}+\left(\mathrm{F}_{(3,107,118,05)}=0.91, \mathrm{p}=0.44, \eta_{\mathrm{p}}{ }^{2}=0.02\right)$ and $\mathrm{CS}-\left(\mathrm{F}_{(3,56,118,05)}=0.20\right.$, $\left.550 \mathrm{p}=0.67, \eta_{\mathrm{p}}{ }^{2}=0.01\right)$. We ran also the analysis twice with and without adjustment for the same seven predictors as adopted for the test phase: positive affect, negative affect, 
anxiety, catastrophising, pain rating obtained during calibration, sex and BMI. We found

a significant main effect of anxiety for $\mathrm{CS}+\left(\mathrm{F}_{(1,24)}=4.35, \mathrm{p}=0.05, \eta_{\mathrm{p}}{ }^{2}=0.15\right)$, however,

this effect did not change the mean effect of Group on mean Pain Expectancy for CS+ during extinction.

556 We also ran separate repeated measures ANOVA for the Precise and the Imprecise group

557 data. These analyses demonstrated extinction of Pain Expectancy ratings for the Precise group at $\mathrm{CS}+\left(\mathrm{F}_{(2,500,47,497)}=8.92, \mathrm{p}<0.01, \eta_{\mathrm{p}}{ }^{2}=0.32\right)$ and $\mathrm{CS}-\left(\mathrm{F}_{(3,545,67,532)}=4.63, \mathrm{p}<0.01\right.$,

$\left.\eta_{\mathrm{p}}{ }^{2}=0.20\right)$ and for the Imprecise group at $\mathrm{CS}+\left(\mathrm{F}_{(3,532,67,104)}=3.92, \mathrm{p}<0.01, \eta_{\mathrm{p}}{ }^{2}=0.18\right)$.

560 Pairwise comparisons showed that for the Precise group, Pain Expectancy at the CS + had

561 lowered by the $5^{\text {th }}$ extinction trial and for the Imprecise group, Pain Expectancy had not lowered by the final extinction trial (Table 4). The Pain Expectancy across trials for each group during extinction phase shows a gradual decrease with subsequent trial (Table 4).

Manipulation check - evidence of classical conditioning

As a confirmation of the classical conditioning effect, we expected a main effect of

567 Stimulus type (higher expectation for CS+ than CS-) during test phase. Higher Pain Expectancy for CS+ than CS- was observed on both groups (Precise group $-\mathrm{F}_{(1,19)}=6.31$, $\mathrm{p}=0.02, \eta_{\mathrm{p}}^{2}=0.12$, Imprecise Group $\left.-\mathrm{F}_{(1,19)}=25.92, \mathrm{p}<0.01, \eta_{\mathrm{p}}{ }^{2}=0.57\right)$. Pairwise comparisons showed a significant mean difference between CS+ and CS- of 3.9 (95\% CI: $1.8-6.0, \mathrm{p}<0.01)$ for the Imprecise group and $2.15(95 \% \mathrm{CI}: 0.2-4.0, \mathrm{p}=0.02)$ for the Precise group.

575 For both groups, we classified a participant as having been contingency aware if they 576 identified either CS+ or GS1 as the location in which they had received painful 
577 stimulation. In the Precise group, 8 participants (40\%) accurately reported the CS+ as the

578 location in which they had received noxious stimulation. Of these 8 participants, four 579 responded to this question by reporting two locations, one of which was either the CS+ 580 or GS1. In the Imprecise group, 3 participants (15\%) accurately reported the CS+ as the

581 location in which they had received painful stimulation.

582 The assessment of awareness of the visual manipulation showed that only two participants

583 (one from each group) disagreed with the statement "I felt as if I was looking at my own 584 hand" during the procedure. However, $21 \%(\mathrm{n}=4)$ from the Precise group and $40 \%(\mathrm{n}=8)$

585 from the Imprecise group agreed that “...something was wrong during some movements”, 586 and $11 \%$ of the participants from the Precise group and $20 \%$ of the Imprecise group 587 agreed that there had been some "incongruence between visual information and the actual 588 position of the hand" during some movements.

\section{Discussion}

591 The main objective of this study was to investigate whether imprecise sensory feedback 592 increases conditioned pain expectancy, and generalization and extinction of that 593 conditioned effect, when a specific location is used as the CS+. Our results supported our 594 primary hypothesis that imprecise sensory input during conditioning would result in 595 greater pain expectancy at the CS+ location. That is, pain expectancy was significant 596 increased when the hand was at a location previously associated with pain when visual 597 feedback had been disrupted via illusion than it was when visual feedback had not been 598 disrupted. Our results also showed generalization of pain expectancy but, contrary to the 599 first of our secondary hypotheses, generalization was not affected by rendering sensory 600 input imprecise during conditioning. Our final hypothesis was not supported either - we 601 detected no difference between groups in the rate at which extinction of the elevated pain 
602 expectancies occurred. Post-hoc separate analysis on group-specific data raise the 603 possibility that an effect was present and we were not powered to detect it.

604 The main finding of the current study was that rendering sensory input imprecise 605 increased pain expectancy at the CS+ location. To put this finding into context, it is 606 important to consider it in light of the broader context of the $\mathrm{IH}^{60}$. The $\mathrm{IH}$ appreciates 607 that, just as visual stimuli are encoded as meaningful singular percepts, not as an array of 608 features or simply a retinal 'impression' ${ }^{41}$ (which allows us to be tricked by illusions such 609 as the Necker cube ${ }^{61}$ ), so too painful movements and events are encoded as meaningful 610 singular multisensory events, not as an array of nociceptive and non-nociceptive sensory

611 features or simply a nociceptive 'impression'. Such high-order integrated percepts present 612 an excellent situation for associative learning, which permits rapid protective responses.

613 That the current experiment induced elevated pain expectancies associated with a given 614 location supports that principle. A mean difference of 1.47 on an 11-point pain 615 expectancy rating should be considered a small effect, although it is comparable in 616 magnitude with previously reported differences between patients and healthy controls ${ }^{48}$ 617 and may well be clinically important - further work is clearly required to determine if 618 this is the case.

619 That imprecise sensory input increased pain expectancies extends a previous result from 620 our group in which differential learning between CS+ vs. GSs and CS- locations in a skin 621 area with low tactile precision (the lower back), was poorer than it was in a skin area with 622 high tactile precision (the hand) ${ }^{26}$. Notably however, the current research question was 623 different in two critical ways. First, we aimed to define a specific location of the hand in 624 space as the CS+. That spatial data can influence the learning of associations was 625 established in early studies ${ }^{72,73}$, and associating certain environments with aversive 626 stimuli is a well-used paradigm to study fear conditioning and learned helplessness in 
627 rats $^{34}$. Moreover, the notion that spatial cues might sufficiently signal threat so as to

628 modulate pain expectancies is well recognized clinically ${ }^{45}$.

629 An important consideration is whether or not our manipulation to induce sensory

630 imprecision may have had other pain-relevant effects unrelated to the conditioning

631 procedure. Sensorimotor conflicts may deflagrate sensory disturbances in chronic pain

632 patients $^{7}$ or exacerbate symptoms ${ }^{14}$. Also, experimentally induced pain has been

633 associated with increased report of sensory disturbances in healthy volunteers, but the

634 increase in sensory disturbances was not explained by an interaction between stimulation

635 and sensorimotor incongruence ${ }^{7}$. Another consideration relates to previous findings that

636 showed that lower stimulus predictability of pain is associated with higher reports of pain,

637 fear and greater physiological arousal ${ }^{8,}{ }^{62}$. We cannot exclude the possibility that

638 processes similar to those interrogated in those studies were also at play here.

639 This approach (combining sensorimotor conflict + unpredictability) clearly has ecological

640 limitations - there is no suggestion that the reliability of visual input is compromised

641 during painful events outside of the laboratory. However, we selected this approach

642 because we wanted to disrupt the final encoding of location and we can be sure, based on

643 our previous work using the MIRAGE system, that we can achieve this aim ${ }^{4,5,20}$. The

644 evaluation of participants' awareness of the visual input manipulation also confirmed that

645 there was approximately a two-fold increase in reports of perceived incongruence

646 between real and seen hand position in the Imprecise versus the Precise group, supporting

647 the idea that participants would likely be less certain and/or accurate in localizing their

648 hand under imprecise feedback.

649 In addition, we investigated the effect of several confounding variables on the pain

650 expectancy. Our results showed just an effect of anxiety on pain expectancy during 651 extinction phase, although, no interaction between anxiety and Group was observed, 
652 suggesting anxiety, prior to the experiment, influenced both groups equally. On the other

653 hand, we did not gather data regarding anxiety or arousal during the experiment. Future

654 studies may investigate the pain anxiety evoked by contexts with different levels of

655 predictability and sensorimotor conflict.

656 Regardless of the sensorimotor conflict and unpredictability, our paradigm was effective

657 in inducing location specific expectancies - differential learning with higher pain 658 expectancy at CS+ than at CS-. However, we did not reliably induce contingency 659 awareness. Just $40 \%$ of participants from the Precise group and $15 \%$ from the Imprecise 660 group, could accurately recall the location at which they received painful stimulation 661 during acquisition phase. That is, more of the Precise group showed contingency 662 awareness in post-experiment questioning, but $60 \%$ of that group still remained unaware.

663 The $50 \%$ reinforcement rate of the association between CS+UCS during acquisition phase 664 may explain low contingency awareness, but not the between-group difference. In fact, 665 such a low rate of contingency awareness with such a clear conditioning effect suggests 666 that conditioning occurred outside of awareness. Whether or not this is possible is a long667 standing debate - the first studies reporting fear conditioning outside of awareness 668 emerged over 75 years ago [e.g. Diven ${ }^{15}$, Haggard ${ }^{23}$ ] and studies reporting otherwise 669 emerged a decade later, clearly linking both conditioning and generalization to both 670 contingency awareness and ability to articulate it [e.g. Chatterjee and Eriksen ${ }^{10}$ ].

671 The current experiment demonstrated that once a pain expectancy was established during 672 'acquisition', the expectancy was elicited also by events that shared some features with 673 it, a process called generalization. In conditioning experiments, generalisation is inversely 674 related to the degree to which a stimulus can be differentiated from other functionally 675 distinct stimuli, which allows optimisation of behavioural specificity ${ }^{22,65}$. Contrary to our 676 prediction, which was based on the $\mathrm{IH}$, generalization of the conditioned response did not 
677 increase, nor did the speed of extinction, when acquisition occurred under imprecise

678 sensory input. Perhaps the alterations in the task during test phase limited differential 679 generalization. That is, generalization was investigated using locations (GSs) outside of 680 the learned movement paths, which may reduce generalization. In addition, CS-UCS 681 reinforcement of $50 \%$ during acquisition phase, (i.e. $50 \%$ of the occasions on which 682 participants crossed the CS+ location they did not receive a stimulus) may have reduced contingency awareness and perhaps conditioning effects and between group differences.

684 However, a previous study showed that despite continuous CS-UCS pairings during 685 acquisition showed stronger conditioned responses, it speeds the rate of extinction of differential UCS expectancies in a study of human fear conditioning ${ }^{21}$. underpowered for analysing all three hypotheses - that our secondary hypotheses were unsupported does not exclude the possibility that an effect exists. Observation of the data and planned pairwise comparisons raise that possibility particularly for our second secondary hypothesis - pain expectancies during extinction were lower after the $4^{\text {th }}$ trial 692 in the Precise group but were still not lower after the $6^{\text {th }}$ trial in the Imprecise group. A 693 second limitation was that the researchers in the current study were not blinded to group 694 allocation because they had to administer the shock and the illusion. That our secondary hypotheses were not upheld suggests this did not impact results, but nonetheless it 696 represents a shortcoming of the study. Third, only healthy subjects were enrolled in the 697 current study, so results are not generalizable to clinical populations and it seems possible 698 that differences may exist in contingency learning and generalization gradients between 699 those with and without chronic pain ${ }^{27,49,51}$. Fourth, we were interested in pain expectancy, 700 but we are unable to exclude the possibility that we inadvertently modulated fear of pain. 701 We considered that within the current design, any change in fear of pain would be 
702 secondary to changes in the expected likelihood of pain and saw no theoretical

703 justification for why either condition would be associated with more or less fear of pain

704 aside from that mediated by expectation. Future studies would be well served to evaluate

705 fear of pain in order to verify these assumptions. Fifth, the CS-UCS reinforcement of 50\%

706 adopted in our study may not have been ideal - continuous and partial CS-UCS pairings

707 can result in different outcomes. Finally, we did not lodge and lock our protocol and

708 statistical analysis plan prior to data collection. When we commenced this study, such

709 practice was uncommon in our field, but now it is recommended, and our group is among

710 those at the forefront of this push ${ }^{36}$. Failure to do this clearly represents a shortcoming in

711 transparency and reporting.

712

\section{Conclusion}

714 Our results supported our primary hypothesis that imprecise sensory input during

715 conditioning would result in a small but increased pain expectancy at the CS+ location,

716 even though most participants, particularly those in the Imprecise group, remained

717 contingency unaware. Such a result adds to a growing body of literature centered around

718 the Imprecision Hypothesis and to evidence for contingency unaware conditioning in pain

719 conditioning studies. We also showed generalization of pain expectancy but, contrary to

720 our hypothesis, neither generalization nor extinction were affected by rendering sensory

721 input imprecise during conditioning. That a post-hoc analysis raised the possibility that

722 an effect of imprecise sensory input on extinction may have gone undetected suggests

723 appropriately powered studies to thoroughly test that hypothesis might be warranted.

724 Future studies should also determine whether the results hold when participants are

725 contingency aware and whether pain modulation is affected in a similar way to pain

726 expectancy modulation. 


\section{References}

729

730

731

732

733

734

735

736

737

738

739

740

741

742

743

744

745

746

747

748

749

1. Acerra NE, Moseley GL. Dysynchiria: watching the mirror image of the unaffected limb elicits pain on the affected side. Neurology 65: 751-3, 2005

2. Arntz A, Claassens L. The meaning of pain influences its experienced intensity. Pain $109: 20-25,2004$

3. Bajcar EA, Adamczyk WM, Wiercioch-Kuzianik K, Bąbel P. Nocebo hyperalgesia can be induced by classical conditioning without involvement of expectancy. PLoS One 15:e0232108, 2020

4. Bellan V, Gilpin HR, Stanton TR, Dagsdóttir LK, Gallace A, Lorimer Moseley G. Relative contributions of spatial weighting, explicit knowledge and proprioception to hand localisation during positional ambiguity. Exp Brain Res 235:447-455, 2017

5. Bellan V, Gilpin HR, Stanton TR, Newport R, Gallace A, Moseley GL. Untangling visual and proprioceptive contributions to hand localisation over time. Exp Brain Res 233:1689-1701, 2015

6. Bland, JM, Altman DG. Measurement Error. Br Med J 313:744-753, 1996

7. Brun C, Mercier C, Grieve S, Palmer S, Bailey J, McCabe CS. Sensory disturbances induced by sensorimotor conflicts are higher in complex regional pain syndrome and fibromyalgia compared to arthritis and healthy people, and positively relate to pain intensity. Eur J Pain 23:483-494, 2019

8. Carlsson K, Andersson J, Petrovic P, Petersson KM, Ohman A, Ingvar M. Predictability modulates the affective and sensory-discriminative neural processing of pain. Neuroimage 32:1804-14, 2006 
9. Catley MJ, O'Connell NE, Berryman C, Ayhan FF, Moseley GL. Is tactile acuity altered in people with chronic pain? a systematic review and meta-analysis. J Pain 15: $985-1000,2014$

10. Chatterjee BB, Eriksen CW. Conditioning and generalization of GSR as a function of awareness. J Abnorm Psychol 60:396-403, 1960

11. Crawford JR, Henry JD. The Positive and Negative Affect Schedule (PANAS): Construct validity, measurement properties and normative data in a large non-clinical sample. Br J Clin Psychol 43: 245-265, 2004

12. Cressman EK, Henriques DY. Motor adaptation and proprioceptive recalibration. Prog Brain Res 191:91-9, 2011

13. Crombez G, Eccleston C, Van Damme S, Vlaeyen JW, Karoly P. Fear-avoidance model of chronic pain: the next generation. Clin J Pain 28: 475-83, 2012

14. Daenen L, Nijs J, Roussel N, Wouters K, Van Loo M, Cras P. Sensorimotor incongruence exacerbates symptoms in patients with chronic whiplash associated disorders: an experimental study. Rheumatology (Oxford) 51(8):1492-9, 2012

15. Diven K. Certain Determinants in the Conditioning of Anxiety Reactions. J Psychol 766 3: $291-308,1937$

16. Edwards RR, Campbell CM, Fillingim RB. Catastrophizing and experimental pain sensitivity: only in vivo reports of catastrophic cognitions correlate with pain responses. J Pain 6:338-339, 2005

17. Engel GL. The clinical application of the biopsychosocial model. Am J Psychiatry $137: 535-44,1980$

18. Gaskin DJ, Richard P. The Economic Costs of Pain in the United States. In: Institute of Medicine (US) Committee on Advancing Pain Research, Care, and Education. Relieving Pain in America: A Blueprint for Transforming Prevention, Care, 
Education, and Research. Washington (DC): National Academies Press (US); 2011. Appendix C. Available from: https://www.ncbi.nlm.nih.gov/books/NBK92521/

777

778

779

780

781

782

783

784

785

786

787

788

789

790

791

792

793

794

795

796

797

798

19. Girden E. R. ANOVA: Repeated Measures. SAGE Publications, 1992

20. Grabherr L, Russek LN, Bellan V, Shohag M, Camfferman D, Moseley GL. The disappearing hand: vestibular stimulation does not improve hand localisation. PeerJ 7:e7201, 2019

21. Grady AK, Bowen KH, Hyde AT, Totsch SK, Knight DC. Effect of continuous and partial reinforcement on the acquisition and extinction of human conditioned fear. Behav Neurosci. 2016;130(1):36-43.

22. Guirlanda S, Enquist MA. A century of generalization. Anim Behav 66:15-36, 2003

23. Haggard EA. Experimental studies in affective processes: I. Some effects of cognitive structure and active participation on certain autonomic reactions during and following experimentally induced stress. J Exp Psychol Gen 33:257-284, 1943

24. Harvie DS, Broecker M, Smith RT, Meulders A, Madden VJ, Moseley GL. Bogus visual feedback alters onset of movement-evoked pain in people with neck pain. Psychol Sci 26:385-92, 2015

25. Harvie DS, Meulders A, Madden VJ, Hillier SL, Peto DK, Brinkworth R, Moseley GL. When touch predicts pain: predictive tactile cues modulate perceived intensity of painful stimulation independent of expectancy. Scand J Pain 11:11-18, 2016a

26. Harvie DS, Meulders A, Reid E, Camfferman D, Brinkworth RS, Moseley GL. Selectivity of conditioned fear of touch is modulated by somatosensory precision. Psychophysiology 53:91-9, 2016b

27. Harvie DS, Moseley GL, Hillier SL, Meulders A. Classical Conditioning Differences Associated With Chronic Pain: A Systematic Review. J Pain 18(8):889-898, 2017 
800

801

802

803

804

805

806

807

808

809

810

811

812

813

814

815

816

817

818

819

820

821

28. Hennings K, Arendt-Nielsen L, Andersen OK. Breakdown of accommodation in nerve: a possible role for persistent sodium current. Theor Biol Med 2:16, 2005

29. Hohwy J. The predictive mind. Oxford: Oxford University Press, 2013

30. Inui K, Tran TD, Hoshiyama M, Kakigi R. Preferential stimulation of Adelta fibers by intra-epidermal needle electrode in humans. Pain 96: 247-252, 2002

31. Jedlicka P. Revisiting the Quantum Brain Hypothesis: Toward Quantum (Neuro)biology?. Front Mol Neurosci 10:366, 2017

32. Jensen K, Kirsch I, Odmalm S, Kaptchuk TJ, Ingvar M. Classical conditioning of analgesic and hyperalgesic pain responses without conscious awareness. Proc Natl Acad Sci USA 112:7863-7, 2015

33. Khan A, Rayner GD. Robustness to non-normality of common tests for the manysample location problem. J Appl Math Decis Sci 7:187-206, 2003

34. Landgraf D, Long J, Der-Avakian A, Streets M, Welsh DK. Dissociation of learned helplessness and fear conditioning in mice: a mouse model of depression. PLoS One 10:e0125892, 2015

35. Landmark T, Romundstad P, Dale O, Borchgrevink PC, Vatten L, Kaasa S. Chronic pain: One-year prevalence and associated characteristics (the HUNT pain study). Scand J Pain 4:182-187, 2013

36. Lee H, Lamb SE, Bagg MK, Toomey E, Cashin AG, Moseley GL. Reproducible and replicable pain research: a critical review. Pain 159: 1683-1689, 2018

37. Loeser JD, Concepts of Pain. In: Stanton-Hicks J, Boaz R, Eds.Chronic Low Back Pain, New York, Raven Press, 1982, pp 145-8

38. Loeser JD, Melzack R. Pain: an overview. Lancet 353:1607-9, 1999 
39. Lovibond PF, Liu JC, Weidemann G, Mitchell CJ. Awareness is necessary for differential trace and delay eyeblink conditioning in humans. Biol Psychol 87:393400,2011

40. Lovibond PF, Shanks DR. The role of awareness in Pavlovian conditioning: empirical evidence and theoretical implications. J Exp Psychol Anim Behav Process. $28: 3-26,2002$

41. Luck SJ, Vogel EK. The capacity of visual working memory for features and conjunctions. Nature 390:279-281, 1997

42. Madden VJ, Bellan V, Russek LN, Camfferman D, Vlaeyen JWS, Moseley GL. Pain by Association? Experimental Modulation of Human Pain Thresholds Using Classical Conditioning. J Pain 17:1105-1115, 2016

43. Madden VJ, Kamerman P, Bellan V, Catley MJ, Russek LN, Camfferman D, Moseley GL. Was that painful or non-painful? The Sensation and Pain Rating Scale (SPARS) performs well in the experimental context. J Pain 20:472.e1-472.e12, 2019

44. Madden VJ, Kamerman PR, Catley MJ, Bellan V, Russek LN, Camfferman D, Moseley GL. Rethinking pain threshold as a zone of uncertainty. bioRxiv 10, 2019. Available at https://www.biorxiv.org/content/10.1101/521302v2.full. Accessed May 28,2020

45. Madden VJ, Moseley GL. Do clinicians think that pain can be a classically conditioned response to a non-noxious stimulus? Man Ther 22:165-73, 2016

46. Madden VJ, Russek LN, Harvie DS, Vlaeyen JWS, Moseley GL. Classical Conditioning Fails to Elicit Allodynia in an Experimental Study with Healthy Humans. Pain Med 18:1314-1325, 2017 
47. Meulders A, Bennett MP. The Concept of Contexts in Pain: Generalization of Contextual Pain-Related Fear Within a de Novo Category of Unique Contexts. J Pain $19: 76-87,2018$

48. Meulders A, Harvie DS, Bowering JK, Caragianis S, Vlaeyen JW, Moseley GL. Contingency learning deficits and generalization in chronic unilateral hand pain patients. J Pain 15(10):1046-56, 2014

49. Meulders A, Harvie DS, Bowering JK, Caragianis S, Vlaeyen JW, Moseley GL. Contingency learning deficits and generalization in chronic unilateral hand pain patients. J Pain 15:1046-56, 2014a

50. Meulders A, Harvie DS, Lorimer Moseley G, Vlaeyen JW. Generalization of PainRelated Fear Using a Left-Right Hand Judgment Conditioning Task Behav Ther

51. Meulders A, Jans A, Vlaeyen JW. Differences in pain-related fear acquisition and generalization: an experimental study comparing patients with fibromyalgia and

52. Meulders A, Meulders M, Vlaeyen JW. Positive affect protects against deficient safety learning during extinction of fear of movement-related pain in healthy individuals scoring relatively high on trait anxiety. J Pain 15:632-44, 2014b

53. Meulders A, Vandebroek N, Vervliet B, Vlaeyen JW. Generalization gradients in cued and contextual pain-related fear: an experimental study in healthy participants.

54. Meulders A, Vlaeyen JW. Reduction of fear of movement-related pain and painrelated anxiety: An associative learning approach using a voluntary movement paradigm. Pain 153:1504-13, 2012 
55. Moseley GL, Arntz A. The context of a noxious stimulus affects the pain it evokes. Pain 133:64-71, 2007

56. Moseley GL, Butler D. Explain Pain Supercharged. Adelaide, Neuro Orthopaedic Institute Group, 2017

57. Moseley GL, Butler DS. Fifteen Years of Explaining Pain: The Past, Present, and Future. J Pain 16:807-13, 2015

58. Moseley GL, Flor H. Targeting cortical representations in the treatment of chronic pain: a review. Neurorehabil Neural Repair 26:646-52, 2012

59. Moseley GL, Parsons TJ, Spence C. Visual distortion of a limb modulates the pain and swelling evoked by movement. Curr Biol 18(22):R1047-R1048, 2008

60. Moseley GL, Vlaeyen JW. Beyond nociception: the imprecision hypothesis of chronic pain. Pain 2015, 156:35-8

61. Necker L. Observations on some remarkable phenomena seen in Switzerland; and on an optical phenomenon which occurs on viewing a figure of a crystal or geometrical solid. Philos Mag 1:329-337, 183

62. Oka S, Chapman CR, Kim B, Shimizu O, Noma N, Takeichi O, Imamura Y, Oi Y. Predictability of painful stimulation modulates subjective and physiological responses. J Pain 11:239-46, 2010

63. Oldfield RC. The assessment and analysis of handedness: the Edinburgh inventory. Neuropsychologia 9:97-113, 1971

64. Ongaro G, Kaptchuk TJ. Symptom perception, placebo effects, and the Bayesian brain. Pain. 2019;160(1):1-4.

891 65. Pavlov I. Lectures on Conditioned reflexes. New York, Liverlight Publishing 892 Company, 1928 
66. Ratcliffe N, Newport R. The Effect of Visual, Spatial and Temporal Manipulations on Embodiment and Action. Front Hum Neurosci 11:227, 2017.

67. Spielberger CD, Gorsuch RL, Lushene R, Vagg PR, Jacobs GA. Manual for the State-Trait Anxiety Inventory. Palo Alto, CA: Consulting Psychologists Press, 1983

68. Stanton TR, Leake HB, Chalmers KJ, Moseley GL. Evidence of Impaired Proprioception in Chronic, Idiopathic Neck Pain: Systematic Review and MetaAnalysis. Phys Ther 96:876-87, 2016

69. Struyf D, Zaman J, Vervliet B, Van Diest I. Perceptual discrimination in fear generalization: Mechanistic and clinical implications. Neurosci Biobehav Rev $59: 201-7,2015$

70. Sullivan MJ, Bishop SR, Pivik J. The Pain Catastrophizing Scale: development and validation. Psychol Assess 7: 524-532, 1995

71. Talbot KA. Discrimination, conditioning and generalization: Test the assumptions of the imprecision hypothesis of pain. PhD thesis. University of South Australia. 2017. Supervisor: Moseley GL.

72. Testa TJ. Causal relationships and the acquisition of avoidance responses. Psychol Rev 81: 491-505, 1974

73. Testa TJ. Effects of similarity of location and temporal intensity pattern of conditioned and unconditioned stimuli on the acquisition of conditioned suppression in rats. J Exp Psychol Anim Behav Process 1:114-121, 1975

74. Traxler J, Madden VJ, Moseley GL, Vlaeyen JWS. Modulating pain thresholds through classical conditioning. PeerJ 7:e6486, 2019

75. Vlaeyen JW, Linton SJ, Fear-avoidance and its consequences in chronic musculoskeletal pain: a state of the art. Pain 85: 317-32, 2000 
917 76. Vlaeyen JW. Learning to predict and control harmful events: chronic pain and $918 \quad$ conditioning. Pain $1561:$ S86-93, 2015

919 77. Vlaeyen JWS, Kole-Snijders AM, Rotteveel AM, Ruesink R, Heuts PH. The role of 920 fear of movement/(re)injury in pain disability. J Occ Rehab 5:235-52, 1995

921 78. Wallwork SB, Bellan V, Catley MJ, Moseley GL. Neural representations and the 922 cortical body matrix: implications for sports medicine and future directions. $\mathrm{Br} \mathbf{J}$ $923 \quad$ Sports Med 50:990-6, 2016

924 79. Woolf CJ. Central sensitization: uncovering the relation between pain and plasticity. $925 \quad$ Anesthesiology 106:864-7, 2007

926 80. Woolf CJ. Evidence for a central component of post-injury pain hypersensitivity. $927 \quad$ Nature 306:686-8, 1983

928 81. Woolf CJ. What to call the amplification of nociceptive signals in the central nervous 929 system that contribute to widespread pain? Pain 155:1911-2, 2014

930 


\section{Figures}

\section{$1 \mathbf{A}$}

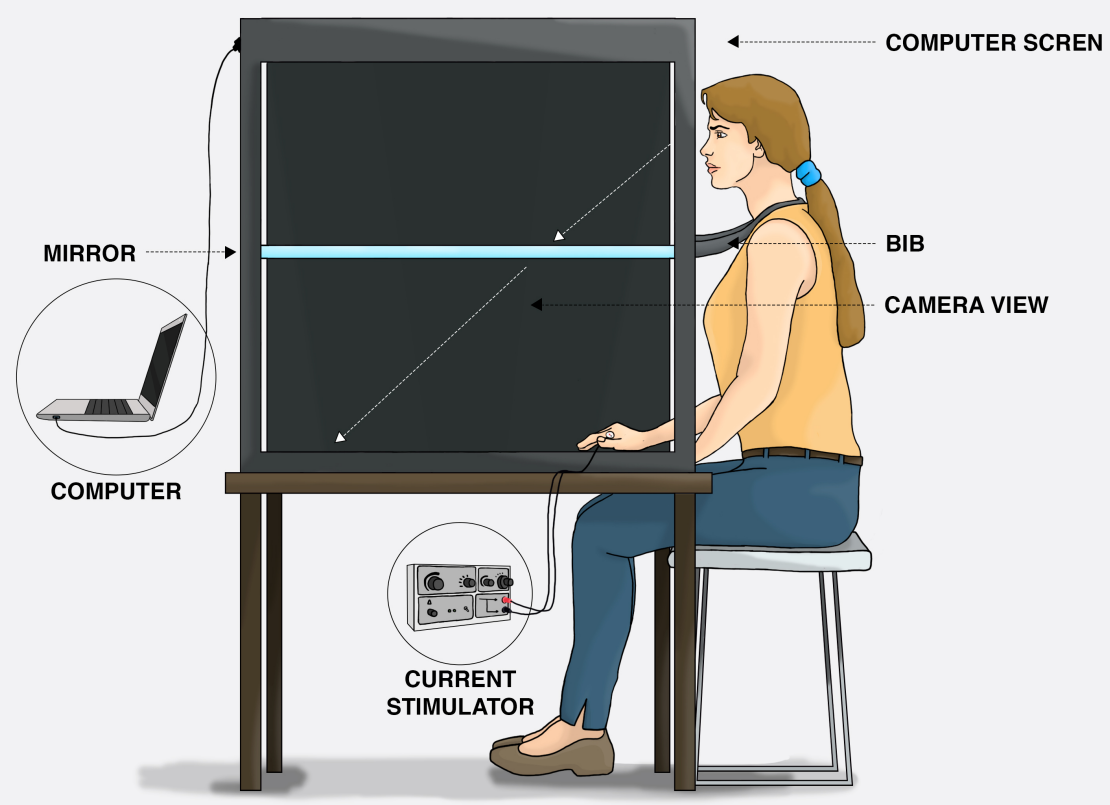

$1 B$

Target I Target 2 Target 3 Target 4 Target 5

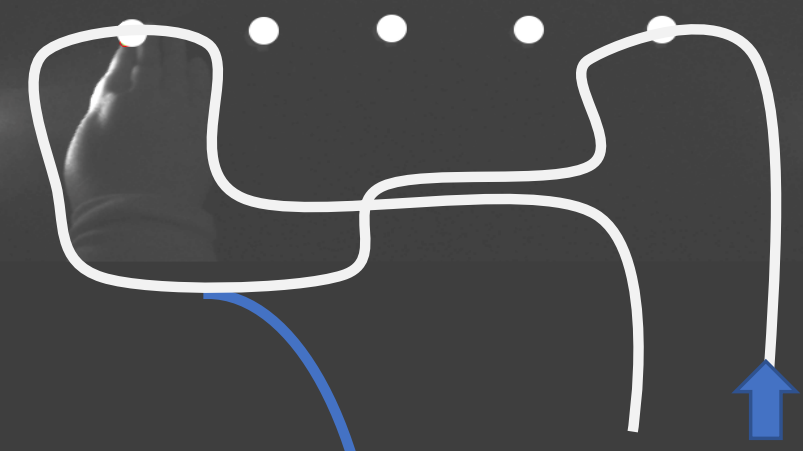

Start position

Target zone

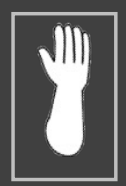

Precise condition : Real position $=$ seen hand position
Target zone

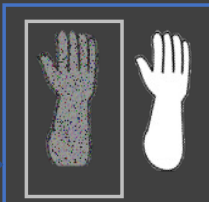

or

Imprecise Condition: Real (blurred hand - inside the target zone) and seen hand position (in white) 
Figure 1A. Schematic representation of the MIRAGE multisensory system. The angles of the camera and mirrors were adjusted to enable real-time video capture of the participants actual hand to be presented in the same spatial position and same visual perspective as if viewing the hand directly.

Figure 1B. (top) Path showing the movement executed during acquisition and extinction phases of the experiment. Participant's hand crossed just the positions related to target 1 and target 5 (conditioned stimulus - CS). This picture is merely illustrative. In other words, no target or path was visible during the acquisition phase, however during test phase the locations were marked with dots [one per trial] and no path was presented. Ultimately, at the beginning of each trial of the extinction phase the same paths performed during acquisition phase, marked with one dot over CS+ or CS- locations were shown to volunteers. The middle positions (Targets 1-3), represent the generalization stimuli locations. Figure 1B. (bottom) The schemas are depicting the real and seen hand locations in the precise condition (right) and imprecise conditions (left) when the participant crossed the target zone (the hand is on the location volunteers received the aversive stimulation). In the example, target 1 was the CS associated with Unconditioned Stimulus (UCS). 
Pay attention to the arrows that are showing the direction

If move your hand to the location how likely do you think is that you will receive a painful stimulation?
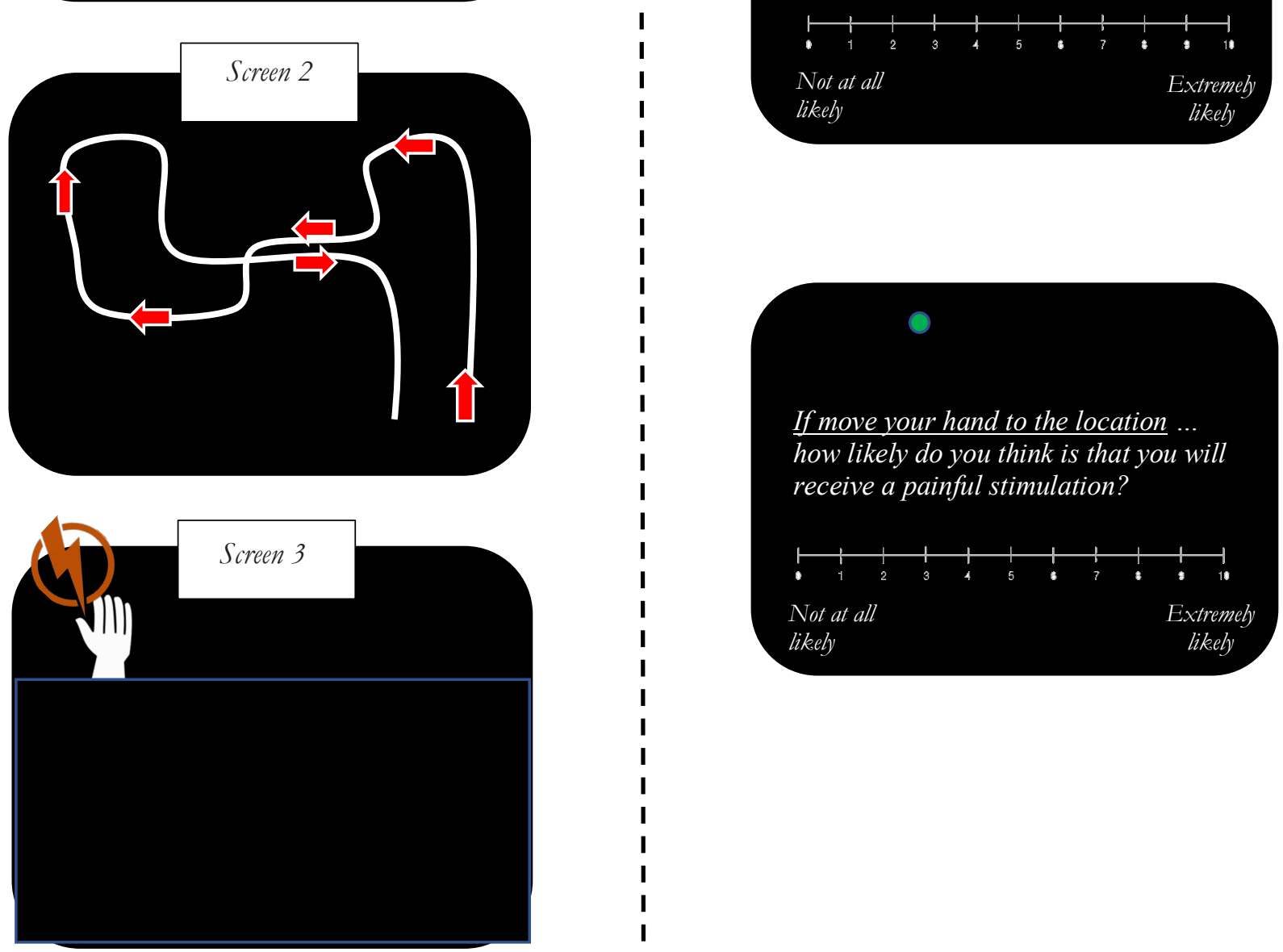

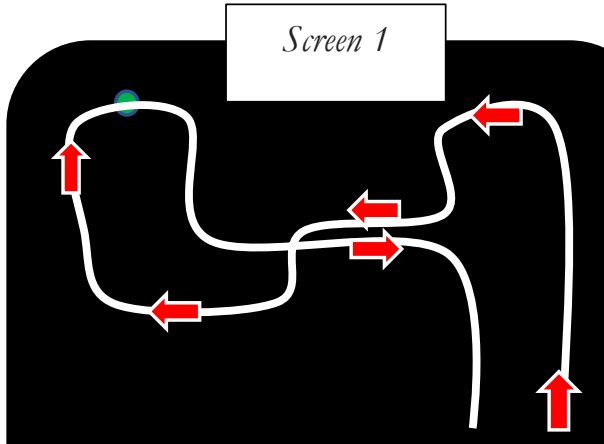

We want you to perform this path Note the location of the dot.

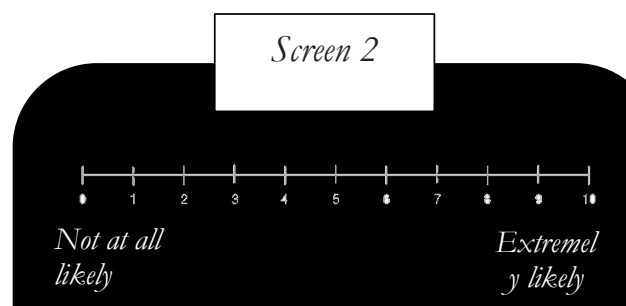

How likely do you think it is, that you will receive a painful stimulation, if you move your hand to that location?

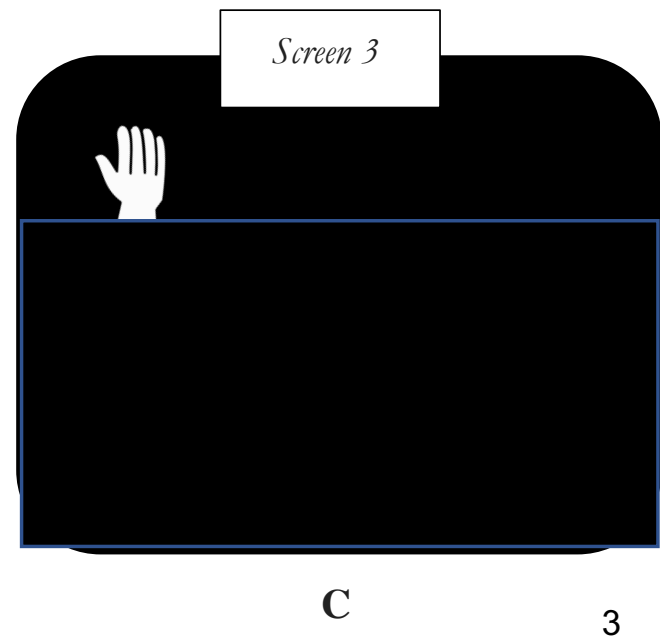


Figure 2B. Flowchart depicting the acquisition phase of the group not submitted to the illusion (precise group). This block is describing the delivery of the conditioned stimulus with noxious stimulation (CS+, target 1). Figure 2B. Flowchart depicting the test phase from the classical conditioning experiment. The screen 1 is describing a trial related to target 1 position and the screen 2 is describing a trial related to target 3. Figure $2 \mathrm{C}$. Flowchart depicting the extinction phase. Screen 1: Screen illustrating the path and the dot related to conditioned stimulus on target 1. Screen 2: Instructions on how to assess pain related expectancy. Screen 3:articipant moving on the path - no noxious stimulation provided. 
Table 1. Descriptive data (mean and standard deviation [SD]) for precise and imprecise groups.

\begin{tabular}{|c|c|c|c|}
\hline Anthropometric and clinical variables & $\begin{array}{l}\text { Precise group } \\
\qquad(\mathrm{n}=\mathbf{2 0})\end{array}$ & $\begin{array}{c}\text { Imprecise group } \\
(n=20)\end{array}$ & p-value \\
\hline Age (years) & $30(12)$ & $26(6)$ & $\mathrm{F}_{(1,38)}=1.80, \mathrm{p}=0.19$ \\
\hline Body Mass Index (BMI) & $25(3)$ & $22(3)$ & $\mathrm{F}_{(1,38)}=5.16, \mathrm{p}=0.03^{*}$ \\
\hline Handedness $(0-100 \%)$ & $80(21)$ & $80(27)$ & $\mathrm{F}_{(1,38)}=0.03, \mathrm{p}=0.86$ \\
\hline Gender ** & $11 \mathrm{~F} / 9 \mathrm{M}$ & $15 \mathrm{~F} / 5 \mathrm{M}$ & $X^{2}=1.75, p=0.32$ \\
\hline Noxious stimulus intensity (mA) & $21(19)$ & $16(14)$ & $\mathrm{F}_{(1,38)}=1.03, \mathrm{p}=0.31$ \\
\hline SPARS $(-50$ to +50$)$ & $28.8(3)$ & $29(3.1)$ & $\mathrm{F}_{(1,38)}=0.22, \mathrm{p}=0.87$ \\
\hline Anxiety (20-80 points) & $37.8(9.3)$ & $36.2(8.3)$ & $\mathrm{F}_{(1,38)}=0.32, \mathrm{p}=0.56$ \\
\hline Positive affect (10-50 points) & $31(7)$ & $30(8)$ & $\mathrm{F}_{(1,38)}=0.05, \mathrm{p}=0.80$ \\
\hline Negative effect (10-50 points) & $17(7)$ & $15(6)$ & $\mathrm{F}_{(1,38)}=0.06, \mathrm{p}=0.41$ \\
\hline Catastrophising (0-24 points) & $6(5)$ & $5(4)$ & $\mathrm{F}_{(1,38)}=0.41, \mathrm{p}=0.53$ \\
\hline \multicolumn{4}{|l|}{$* \mathrm{p}<0.05$ (ANOVA) } \\
\hline \multicolumn{4}{|l|}{$* *$ Chi-square, fisher correction } \\
\hline \multicolumn{4}{|c|}{ Handedness - Edinburgh Handedness Inventory; Anxiety - State Trait anxiety } \\
\hline \multicolumn{4}{|c|}{ Inventory; The Catastrophizing Questionnaire; The Positive and Negative Affect Scale } \\
\hline A & Rati & & \\
\hline
\end{tabular}


Table 2. Pain expectancies at each location for each group. Estimated means for pain related expectancy (mean along the trials) and Standard Deviations (SD) for the precise (P) and imprecise (IMP) groups during test phase

\begin{tabular}{lccc}
\hline & $\begin{array}{c}\text { Estimated mean* (SD) } \\
\text { Precise group }\end{array}$ & $\begin{array}{c}\text { Estimated mean* (SD) } \\
\text { Imprecise group }\end{array}$ & $\begin{array}{c}\text { Mean difference } \\
\text { (IMP-P) }\end{array}$ \\
\hline CS+ & $5.4(2.48)$ & $6.87(1.93)$ & $1.47^{* *}$ \\
GS1 & $4.87(1.48)$ & $5.6(1.84)$ & 0.72 \\
GS2 & $4.04(2.12)$ & $4.75(1.37)$ & 0.71 \\
GS3 & $3.48(2.02)$ & $3.58(1.51)$ & 0.1 \\
CS- & $3.27(2.69)$ & $2.97(2.1)$ & -0.31 \\
\hline
\end{tabular}

*Estimated marginal mean (model precise and imprecise groups vs. conditioned stimuli) $* * \mathrm{p}<0.05$, ANOVA, Bonferroni correction

$\mathrm{CS}+$ : conditioned stimulus associated with noxious stimulation - right or left extreme position, GS1: Generalization stimuli closest to CS+; GS2: middle position; GS3: Generalization stimuli closest to CS- and CS-: the opposite position regarding CS+. 
Table 3. Pain Expectancies at each location for each group during test phase. Estimated mean difference for pairwise comparisons between conditioned and/or generalization stimuli for the Imprecise group (top panel) and Precise group (bottom panel) during test phase.

\begin{tabular}{|c|c|c|c|c|c|}
\hline & $\mathbf{C S}+$ & GS1 & GS2 & GS3 & CS- \\
\hline \multicolumn{6}{|c|}{ Imprecise group (estimated mean difference) } \\
\hline $\mathbf{C S}+$ & NA & - & - & - & - \\
\hline GS1 & -1.27 & NA & - & - & - \\
\hline GS2 & $-2.13^{*}$ & -0.84 & NA & - & - \\
\hline GS3 & $-3.28 *$ & -2 & -1.15 & NA & - \\
\hline CS- & $-3.9 *$ & $-2.63 *$ & -1.77 & -0.62 & NA \\
\hline \multicolumn{6}{|c|}{ Precise group (estimated mean difference) } \\
\hline $\mathbf{C S}+$ & NA & - & - & - & - \\
\hline GS1 & -0.53 & NA & - & - & - \\
\hline GS2 & -1.35 & -0.84 & NA & - & - \\
\hline GS3 & $-2.01 *$ & -1.39 & -0.56 & NA & - \\
\hline CS- & $-2.15^{*}$ & -1.6 & -0.75 & -0.2 & NA \\
\hline
\end{tabular}

*Significant difference (ANOVA, Bonferroni correction, $\mathrm{p}<0.05$ )

$\mathrm{NA}=$ Not applicable, $\mathrm{CS}+=$ conditioned stimulus associated with the noxious stimulus;

$\mathrm{GS}=$ Generalisation stimuli, with GS1 closest to CS+; GS2; CS- unpaired location in the opposite position (and furthest from) the CS+.

Gray cells $=$ repeated comparisons 
Table 4. Pain expectancies during extinction phase. Estimated mean pain expectancies (standard deviations) at the CS+ and CS- locations for the Precise group (left) and Imprecise group (right). Mean values adjusted by anxiety score

\begin{tabular}{lcccc}
\hline & CS- & CS+ & CS- & CS+ \\
\hline \multicolumn{7}{c}{ Precise group (SD) } & Imprecise group (SD) \\
& \multicolumn{2}{c}{ n=20 } & & $\mathbf{n}=\mathbf{2 0}$ \\
\hline Trial 1 & $4.05(2.21)$ & $6.25(2.22)$ & $3.6(2.21)$ & $6.4(1.82)$ \\
Trial 2 & $3.9(2.36)$ & $5.9(1.62)$ & $3.65(2.66)$ & $6.3(1.81)$ \\
Trial 3 & $3.5(1.99)$ & $5.4(1.9)$ & $3.05(2.46)$ & $5.45(2.16)$ \\
Trial 4 & $2.9(2.47)$ & $4.9(2.45)$ & $2.65(2.58)$ & $5.2(2.86)$ \\
Trial 5 & $2.40(1.96)$ & $3.6^{* \# \&}(2.46)$ & $2.6(2.39)$ & $4.95(3.35)$ \\
Trial 6 & $2.25(1.89)$ & $4.0^{\#}(2.71)$ & $2.8(2.65)$ & $4.7(2.9)$ \\
\hline
\end{tabular}

* Different from Trial 1 in pairwise comparisons $(\mathrm{p}<0.05$, Bonferroni)

\# Different from Trial 2 in pairwise comparisons ( $\mathrm{p}<0.05$, Bonferroni)

${ }^{\&}$ Different from Trial 3 in pairwise comparisons $(\mathrm{p}<0.05$, Bonferroni)

$\mathrm{CS}+=$ location that was paired with noxious stimulus during acquisition; $\mathrm{CS}-=$ the location that was unpaired during acquisition and furthest from the CS+. 
Supplementary results Table S8. Description of the mean and standard deviations (SD) of the seen and actual hand position (mm) obtained from pictures captured during acquisition phase of classical conditioning for precise and imprecise groups.

Imprecise Group $\quad$ Precise group

\begin{tabular}{|c|c|c|c|c|c|c|c|}
\hline \multirow[b]{2}{*}{ Target $1-$ left side $(n=10)$} & \multicolumn{3}{|c|}{ Shift to left (SD) } & \multicolumn{3}{|c|}{ Shift to right (SD) } & \multirow[b]{2}{*}{ Mean of 6 pictures } \\
\hline & 50 & 40 & 30 & 30 & 40 & 50 & \\
\hline actual hand (mm) & $158.8(32.5)$ & $149.3(44.8)$ & $132.1(24)$ & $106.4(29.4)$ & $106.5(30.3)$ & $113.3(52.1)$ & $115.1(15.8)$ \\
\hline \multicolumn{8}{|l|}{ Target $5-$ right side $(n=9) * *$} \\
\hline seen hand $(\mathrm{mm})$ & $160.9(34.2)$ & $133.2(37.7)$ & $145(37.9)$ & $108.8(36.8)$ & $123.1(41)$ & $86.6(41.4)$ & $128.4(14.2)$ \\
\hline actual hand (mm) & $112.7(36.4)$ & $93.7(37.4)$ & $111.3(42.6)$ & $133(35.4)$ & $163.7(40.4)$ & $124.3(26.2)$ & $128.4(14.2)$ \\
\hline
\end{tabular}

* Mean value obtained after use the formula for each participant (not the subtraction between mean actual hand - mean seen hand positions)

** During the experiment we missed the pictures from one participant 


\section{SUPPLEMENTARY FILES}

\section{Supplementary Methods}

\section{Supplementary Methods file S1 - Determining the image shift used in the imprecise}

procedure

The amount of shift for the imprecise group was determined during a pilot study $(n=3)$ in which we calculated the position of the tip of the index finger on the right hand (in millimeters - mm) with and without the shift. During the pilot testing, participants were asked to reach dots projected virtually on the board of the MIRAGE box in five positions (Figure S1). Afterwards the dot flashed, participants were instructed to move and reach the position and "to freeze the hand" in the final position. The participants repeated the trials in each position and at different shift ranges and directions (no shift: precise situation, imprecise: shift of $10-50 \mathrm{~mm}$ to right and left) at least five times. For each final position of the hand, the researcher calculated the coordinates using the tip of index finger as a reference with Labview software (Figure S2). The mean value obtained for the coordinate (position on the board in $\mathrm{mm}$ ) for imprecise condition was subtracted from the precise condition, to establish the "real shift", or in other words, the final level of deviation from the correct position.

The standard error of the measurement (SEM) was obtained in an attempt to control the level of "real shift" we provided to participants. To calculate the standard error of the measurement (SEM), we adopted the formula described by Bland and Altman ${ }^{6}$. For the extreme positions, the SEM obtained in the pilot study in the precise condition were: extreme end right position (target 5) $9.71 \mathrm{~mm}$ and extreme end left position (target 1) was $10.32 \mathrm{~mm}$. In order to guarantee that the shifts would surpass the SEM obtained, we adopted a minimum shift of $20 \mathrm{~mm}$ for the study. Values higher than $50 \mathrm{~mm}$ were not considered since the displacement of the screen would be too gross that was possible to 
identify a dark rectangular area on the boundary which may provide cues regarding the manipulation of the image showed and introduce bias. 


\section{Supplementary Methods file S2}

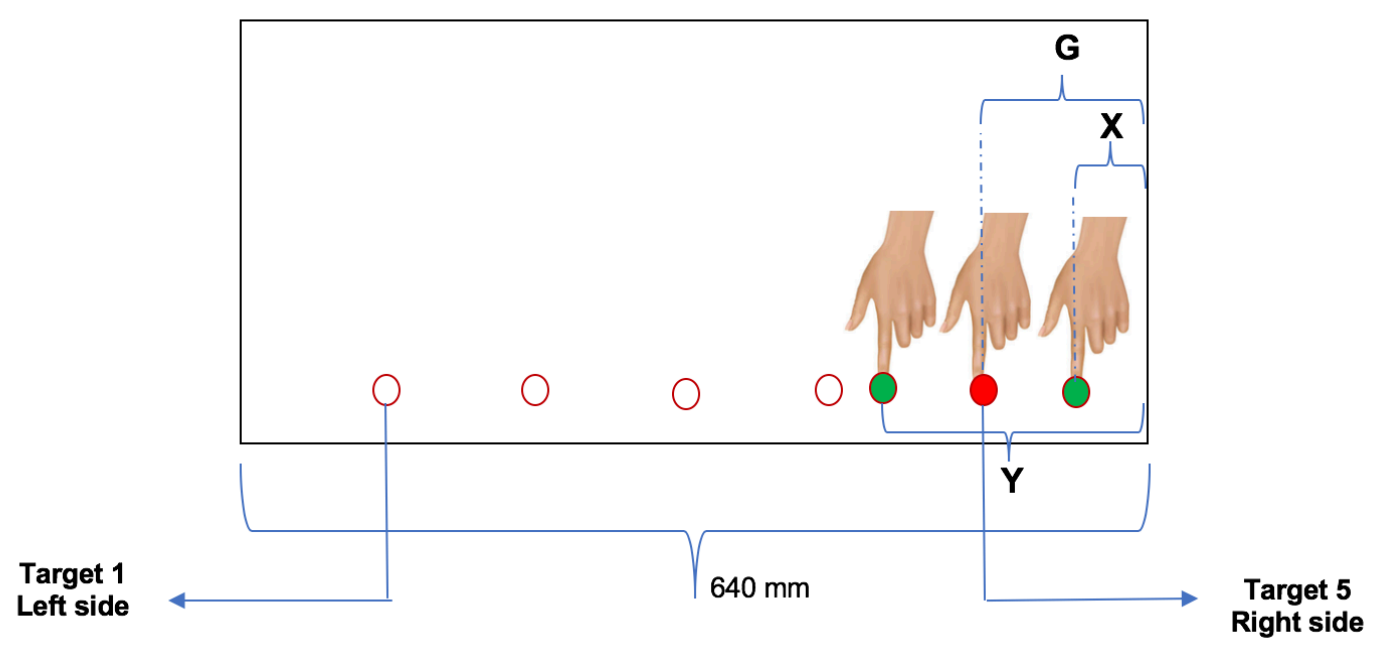

$\mathrm{G}=$ position where the dot flashed - without shift $(119 \mathrm{~mm})$

$X($ shift to right $)=70 \mathrm{~mm}$ and $Y($ shift to left $)=167 \mathrm{~mm}$

Final Shifted $_{\text {position }}-$ Final non-shifted ${ }_{\text {position }}=$ Real Shift

$50 \mathrm{~mm}$ shift to the right: $167-119=\mathbf{4 8 m m}$ of real shift

$50 \mathrm{~mm}$ shift to the left: $70-119=\mathbf{4 9} \mathbf{~ m m}$ of real shift

Figure S2. The picture is showing the calculation to obtain the mean difference for the index finger tip position from imprecise - precise position (Formula: Final Shifted position - Final non-shifted position $=$ Real shift). The illustration depicts position shifted by $5 \mathrm{~cm}$ $(50 \mathrm{~mm})$ to left and right for target 5 (right). 


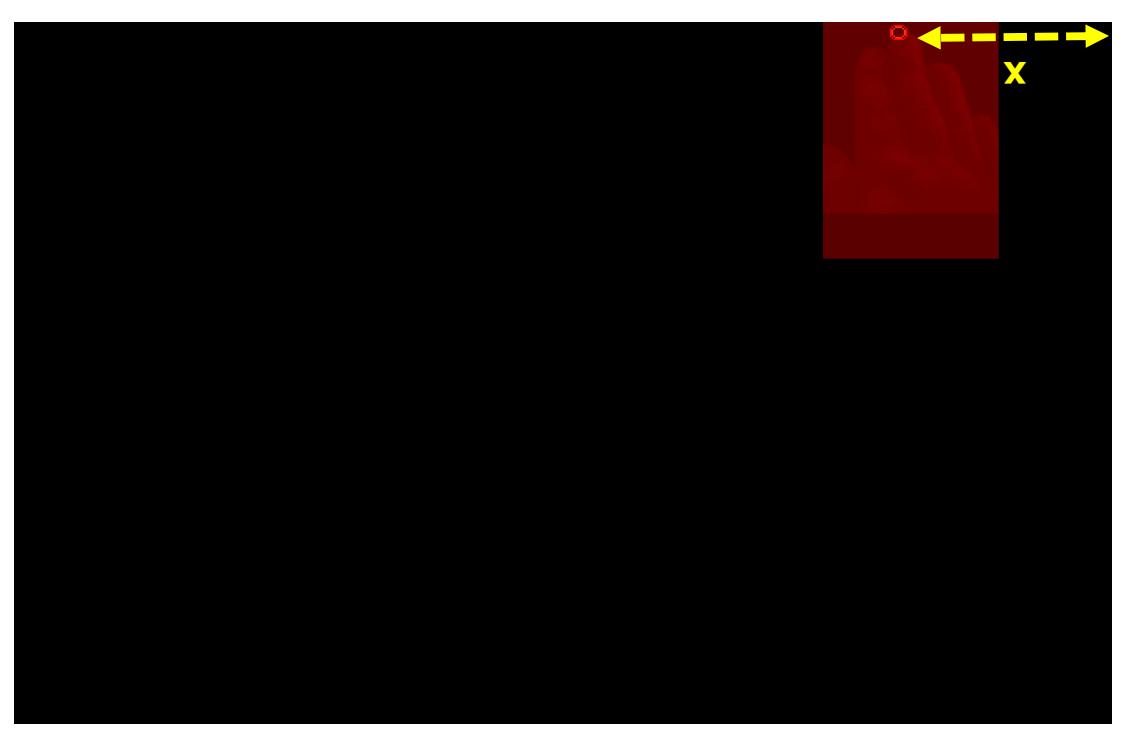

Figure S3a. Illustration depicting the measure of the distance between the target 1 (extreme end - left) and the left border of the screen on the picture. In this picture the seen hand and actual hand are the same (precise context). The distance " $x$ " is $137 \mathrm{~mm}$.

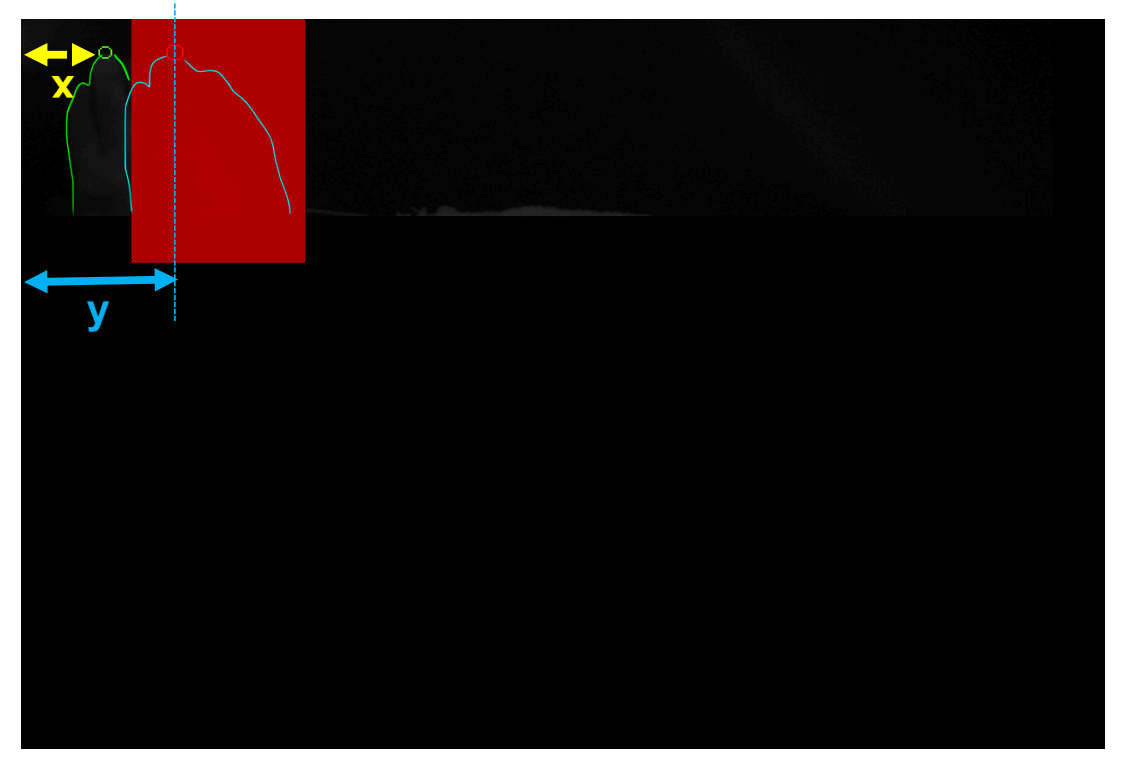

Figure S3b. Illustration depicting the measurement of the distance between the target 5 (extreme end - right) and the right border of the screen on the picture. In this picture the seen hand is the green one and the actual hand is the hand marked with a red dot (imprecise context). It is possible to see the image shifted to the right $(40 \mathrm{~mm})$, considering the participant perspective. The distance " $\mathrm{y}$ " is $126 \mathrm{~mm}$ and the distance $\mathrm{x}$ is $83 \mathrm{~mm}$. 


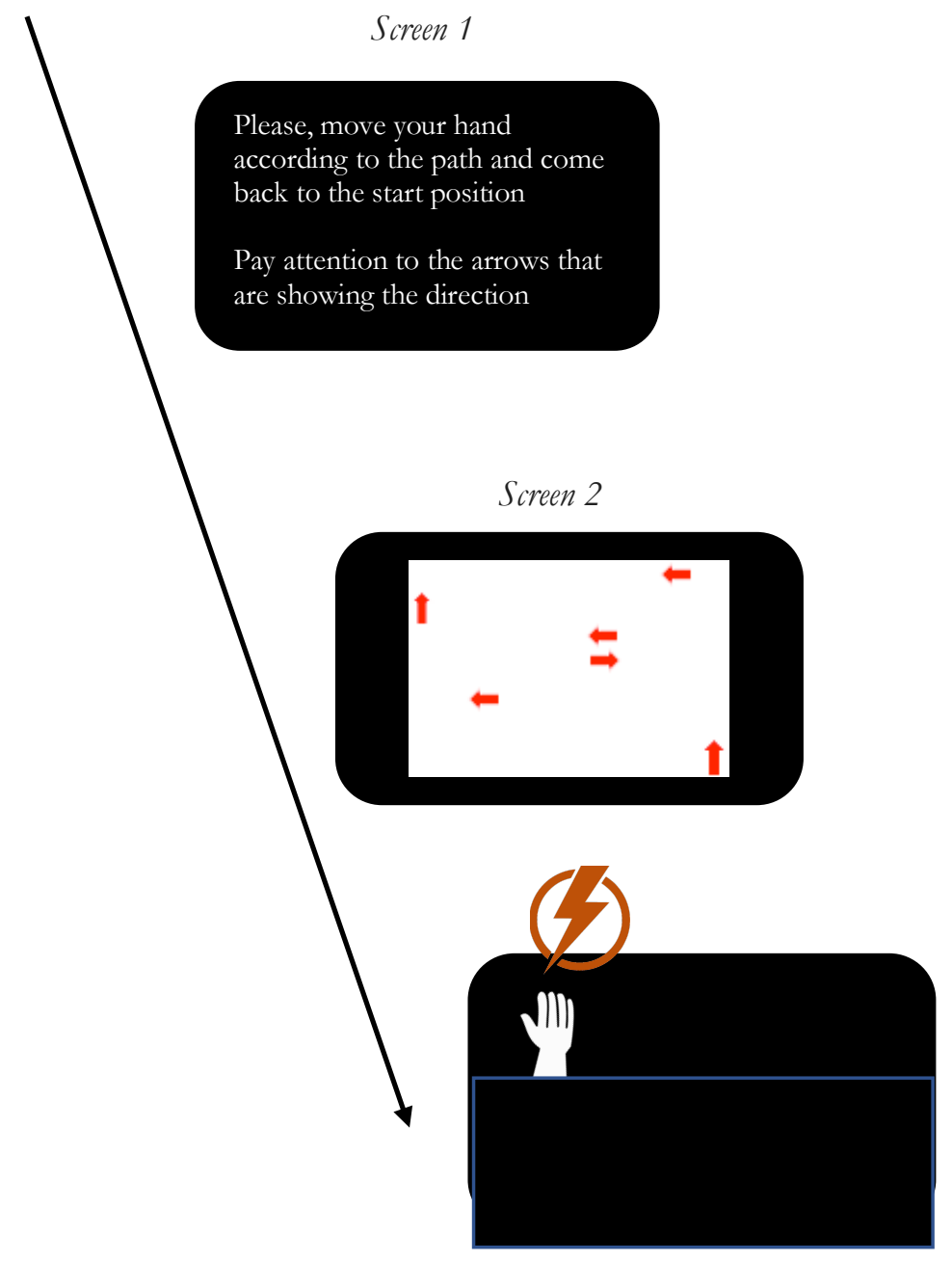

Screen 3-participant view

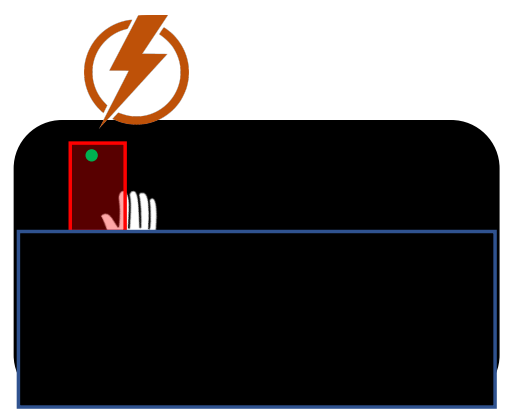

Screen 3 - researcher view

Figure S4. Flowchart depicting the acquisition phase of the group not submitted to the illusion (precise group) showing participant and researcher views. This block is describing the delivery of the conditioned stimulus with high noxious stimulation (CS+, target 1). The blue rectangle was used during the experiment to avoid the participant to update/correct the hand position to match the visual estimate (proprioceptive recalibration). 


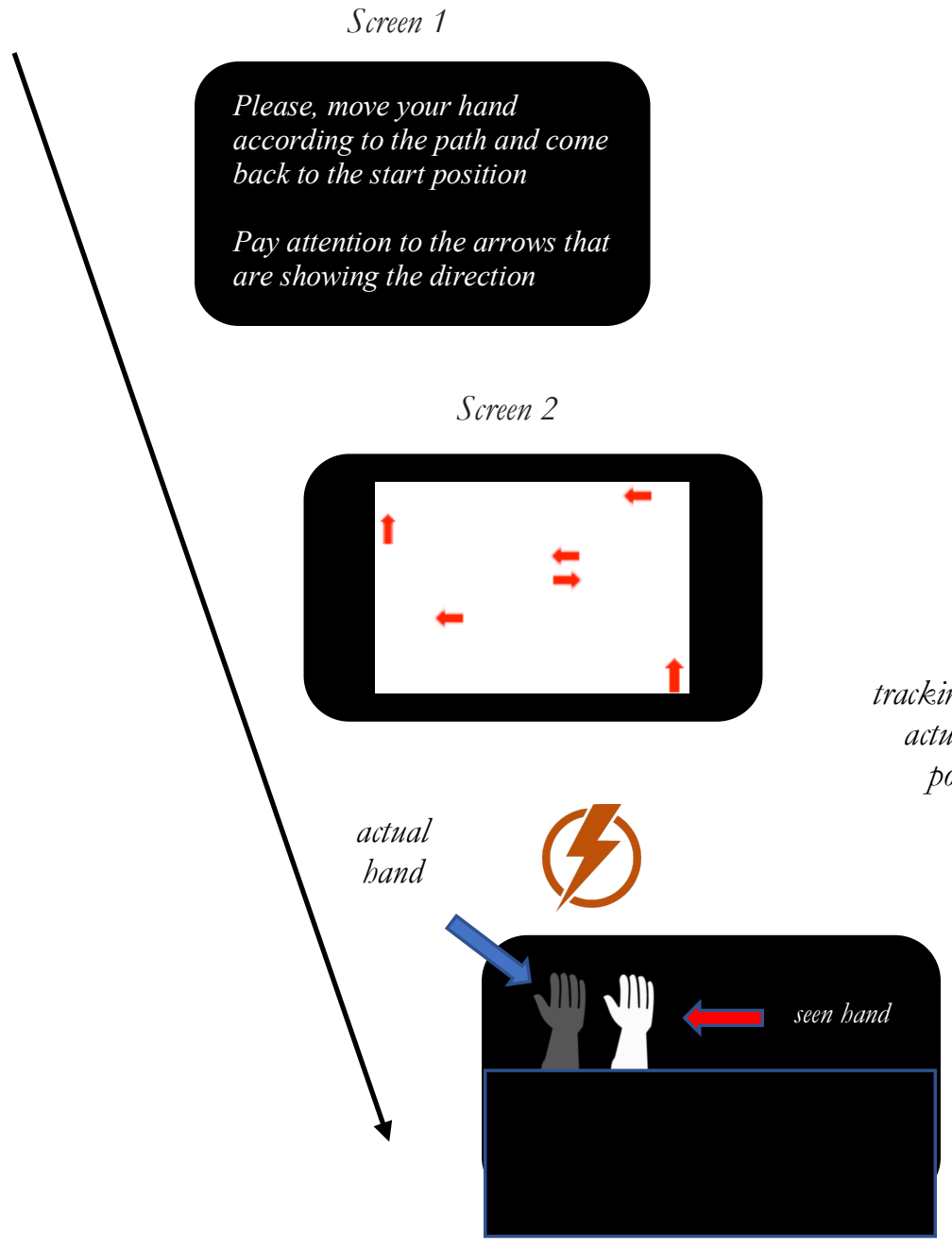

Screen 3-participant view

\section{racking pointing actual hand \\ position}

tracking showing seen band position

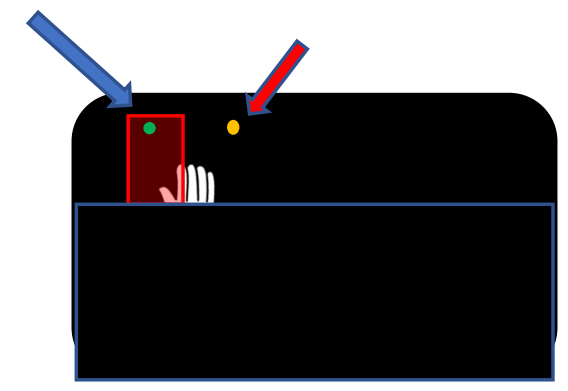

Screen 3 - researcher view

Figure S5. Flowchart depicting the acquisition phase of the group submitted to the illusion (imprecise groups) showing participant and researcher views. This block is describing the delivery of the conditioned stimulus with noxious stimulation $(\mathrm{CS}+$, target 1). The blue rectangle was used during the experiment to avoid the participant to update/correct the hand position to match the visual estimate (proprioceptive recalibration). 


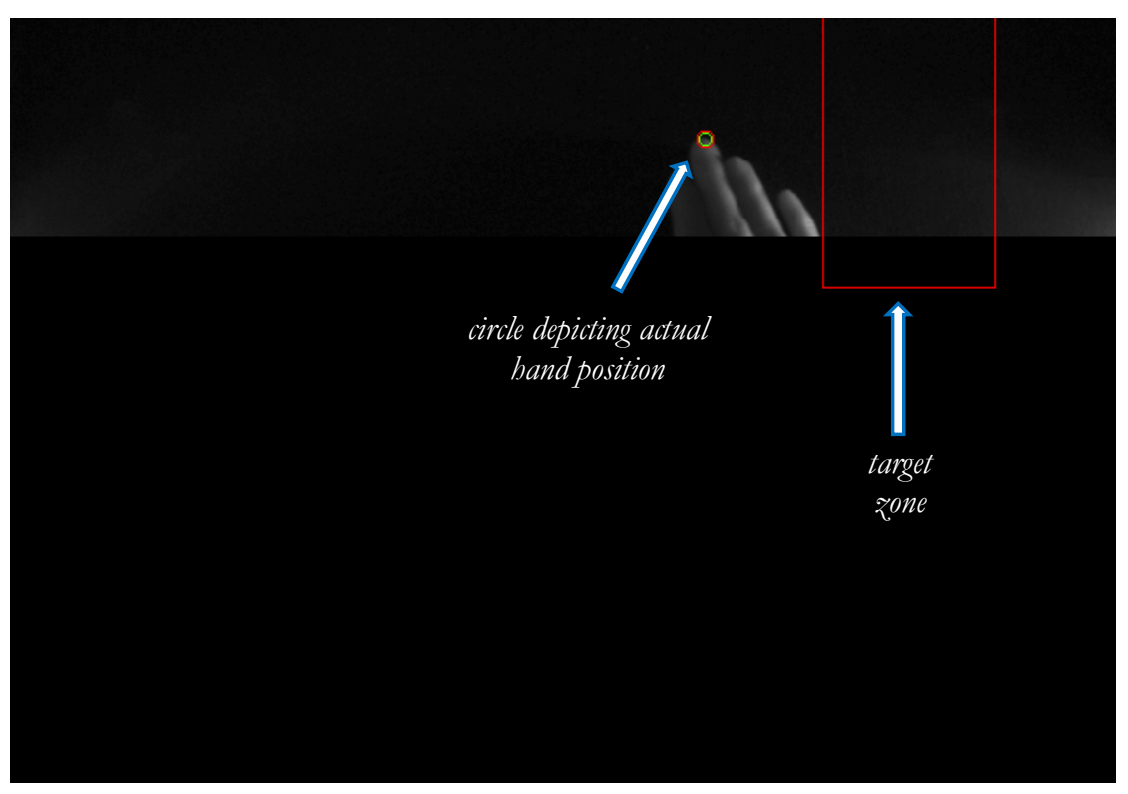

Figure S6a. Picture of the participant moving on the path (left to right) and showing the target zone deactivated, as the hand did not reach the perimeter of the target zone. This view was not available for the participants.

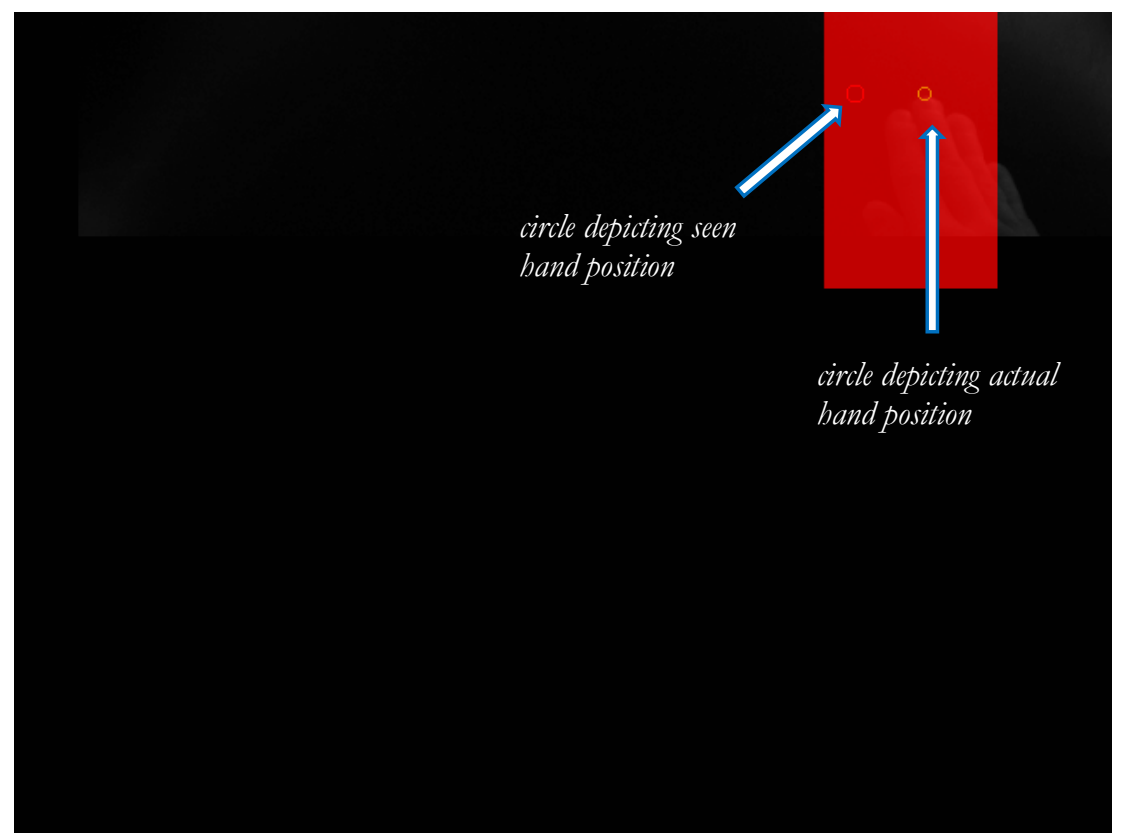

Figure S6b. Picture of the participant moving on the path (left to right) when crossing the position related to conditioned stimulus (in this case target 5) and triggering the target zone. This view was not available for the participants. 
Supplementary Methods file S7.

Five different sequences - Test phase (chosen by lot)

\begin{tabular}{|c|c|c|c|c|c|}
\hline Trial order & Sequence A & Sequence $\mathrm{B}$ & Sequence C & Sequence D & Sequence E \\
\hline 1 & TARGET 1 & TARGET 3 & TARGET 5 & TARGET 4 & TARGET 1 \\
\hline 2 & TARGET 2 & TARGET 1 & TARGET 1 & TARGET 1 & TARGET 3 \\
\hline 3 & TARGET 5 & TARGET 5 & TARGET 3 & TARGET 3 & TARGET 5 \\
\hline 4 & TARGET 4 & TARGET 4 & TARGET 4 & TARGET 5 & TARGET 2 \\
\hline 5 & TARGET 3 & TARGET 2 & TARGET 2 & TARGET 2 & TARGET 4 \\
\hline 6 & TARGET 2 & TARGET 1 & TARGET 2 & TARGET 5 & TARGET 3 \\
\hline 7 & TARGET 5 & TARGET 4 & TARGET 5 & TARGET 3 & TARGET 1 \\
\hline 8 & TARGET 4 & TARGET 3 & TARGET 4 & TARGET 2 & TARGET 2 \\
\hline 9 & TARGET 1 & TARGET 2 & TARGET 1 & TARGET 4 & TARGET 4 \\
\hline 10 & TARGET 3 & TARGET 5 & TARGET 3 & TARGET 1 & TARGET 5 \\
\hline 11 & TARGET 1 & TARGET 4 & TARGET 1 & TARGET 1 & TARGET 5 \\
\hline 12 & TARGET 4 & TARGET 1 & TARGET 3 & TARGET 4 & TARGET 3 \\
\hline 13 & TARGET 3 & TARGET 2 & TARGET 4 & TARGET 2 & TARGET 2 \\
\hline 14 & TARGET 2 & TARGET 5 & TARGET 5 & TARGET 5 & TARGET 4 \\
\hline 15 & TARGET 5 & TARGET 3 & TARGET 2 & TARGET 3 & TARGET 1 \\
\hline 16 & TARGET 4 & TARGET 5 & TARGET 3 & TARGET 3 & TARGET 5 \\
\hline 17 & TARGET 1 & TARGET 1 & TARGET 1 & TARGET 1 & TARGET 1 \\
\hline 18 & TARGET 5 & TARGET 3 & TARGET 5 & TARGET 5 & TARGET 3 \\
\hline 19 & TARGET 2 & TARGET 2 & TARGET 4 & TARGET 4 & TARGET 2 \\
\hline 20 & TARGET 3 & TARGET 4 & TARGET 2 & TARGET 2 & TARGET 4 \\
\hline
\end{tabular}

